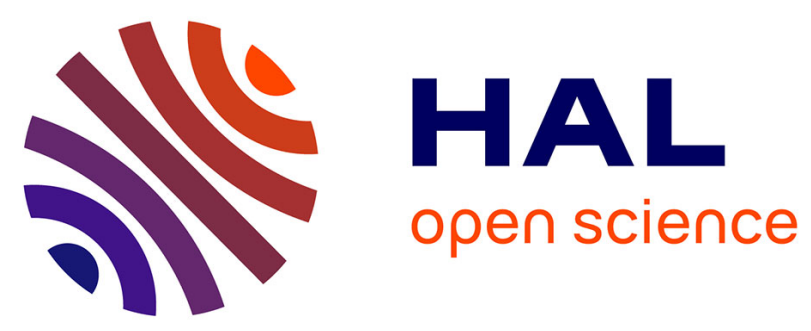

\title{
Private ownership economies with externalities and existence of competitive equilibria: A differentiable approach
}

Elena L. del Mercato, Vincenzo Platino

\section{To cite this version:}

Elena L. del Mercato, Vincenzo Platino. Private ownership economies with externalities and existence of competitive equilibria: A differentiable approach. 2016. halshs-01164015v2

\author{
HAL Id: halshs-01164015 \\ https://shs.hal.science/halshs-01164015v2
}

Submitted on 17 Jan 2017

HAL is a multi-disciplinary open access archive for the deposit and dissemination of scientific research documents, whether they are published or not. The documents may come from teaching and research institutions in France or abroad, or from public or private research centers.
L'archive ouverte pluridisciplinaire HAL, est destinée au dépôt et à la diffusion de documents scientifiques de niveau recherche, publiés ou non, émanant des établissements d'enseignement et de recherche français ou étrangers, des laboratoires publics ou privés. 


\section{Documents de Travail du

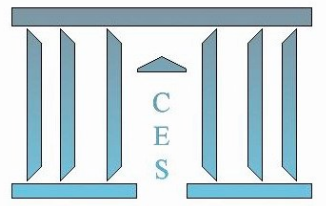

Private ownership economies with externalities and existence of competitive equilibria:

A differentiable approach

Elena L. del Mercato, Vincenzo Platino

2015.34R

Version révisée

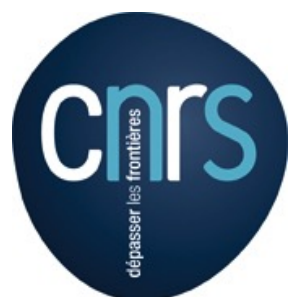




\title{
Private ownership economies with externalities and existence of competitive equilibria: A differentiable approach ${ }^{1}$
}

\author{
This version: December 2016
}

The revised version of the 2016's version has been accepted for publication on Journal of Economics, forthcoming 2017

Elena L. del Mercato and Vincenzo Platino ${ }^{2}$

\begin{abstract}
We consider a general equilibrium model of a private ownership economy with consumption and production externalities. Utility functions and production technologies may be affected by the consumption and production activities of all other agents in the economy. We use homotopy techniques to show that the set of competitive equilibria is non-empty and compact. Fixing the externalities, the assumptions on utility functions and production technologies are standard in a differentiable framework. Competitive equilibria are written in terms of first-order conditions associated with agents' behavior and market clearing conditions, following the seminal paper of Smale (1974). The work of adapting the homotopy approach to economies with externalities on the production side is non trivial and it requires some ingenious adjustments, because the production technologies are not required to be convex with respect to the consumption and production activities of all agents.
\end{abstract}

JEL classification: C62, D51, D62.

Key words: externalities, private ownership economy, competitive equilibrium, homotopy approach. 


\section{Introduction}

We consider a general equilibrium model of a private ownership economy with consumption and production externalities. We use differential techniques to show that the set of competitive equilibria is non-empty and compact.

The general equilibrium model of Arrow and Debreu has been extended to economies with externalities. Our model of externalities is based on the seminal models given in Arrow and Hahn (1971), Laffont and Laroque (1972), and Laffont (1988), where individual preferences and production technologies are affected by the consumption and production activities of all other agents in the economy. Consumption externalities have been widely recognized in the literature on network externalities and other-regarding preferences. Polluting production activities are a classical example of production externalities induced on individual preferences and technological processes of other firms. Furthermore, ICT (Information and Communication Technologies) firms also are in a between-firms externality relationship, because of the influence of the production activities of one firm on another's productivity.

We consider a private ownership economy with a finite number of commodities, households and firms. Each household's preferences are represented by a utility function. Each firm's production technology is represented by a transformation function. ${ }^{3}$ Utility and transformation functions may be affected by the consumption and production activities of all other agents. Each agent (household or firm) maximizes his goal by taking as given both the commodity prices and the choices of every other agent in the economy. The associated

$\overline{1}$ The first version of this paper dates back to 2011 and it has been available on the website: http://www.parisschoolofeconomics.eu/fr/del-mercato-elena/publications. We are delighted that this paper has been quoted by Balasko (2015). Furthermore, we have recently found a similar contribution in Ericson and Kung (2015). The 2011's version has been presented at the Public Economic Theory (PET 10) and Public Goods, Public Projects, Externalities (PGPPE) Closing Conference, Bogazici University, 2010, and the Fifth Economic Behavior and Interaction Models (EBIM) Doctoral Workshop on Economic Theory, Bielefeld University, 2010. We thank the participants of these conferences for useful comments.

2 Elena L. del Mercato, Université Paris 1 Panthéon-Sorbonne, Centre d'Economie de la Sorbonne and Paris School of Economics, address: Centre d'Economie de la Sorbonne, 106-112 Boulevard de l'Hôpital, 75647 Paris Cedex 13, France, email: Elena.delMercato@univ-paris1.fr; Vincenzo Platino (corresponding author), Department of Economics and Statistics, University of Naples Federico II and CSEF, address: Via Cintia Monte S. Angelo, 80126 Napoli, Italy, e-mail: vincenzo.platino@gmail.com.

3 This is a convenient way to represent a production set using an inequality on a function called the transformation function, see for instance Mas-Colell et al. (1995). 
concept of competitive equilibrium is an equilibrium $\grave{a}$ la Nash, the resulting allocation being feasible with the initial resources of the economy. This notion is given, for instance, in Arrow and Hahn (1971), and Laffont (1988), and it includes as a particular case the classical equilibrium definition without externalities.

Fixing the externalities, the assumptions on utility and transformation functions are standard in a differentiable framework. In particular, preferences and production technologies are convex. However, we do not require preferences and production technologies to be convex with respect to the externalities. Our main theorem (Theorem 8) states that the set of competitive equilibria with strictly positive consumptions and strictly positive prices is non-empty and compact. We prove Theorem 8 following the seminal work by Smale (1974), and more recent contributions by Villanacci and Zenginobuz (2005), del Mercato (2006) and Bonnisseau and del Mercato (2008). That is, we use:

(1) Smale's approach.

(2) Homotopy techniques.

(3) The topological degree modulo 2.

In Smale's approach, the equilibrium is written as a solution of an extended system of equations consisting of the first-order conditions associated with agents' behavior and the market clearing conditions. No externalities are taken into account in the seminal work of Smale (1974). However, this approach is used in many different settings such as incomplete markets, public goods and externalities. In the presence of externalities, Smale's approach overcomes the following difficulty: the individual demand and supply depend on the individual demand and supply of all other agents which, in turn, depend on the individual demand and supply of all other agents, and so on. Therefore, it is problematic to define an aggregate demand and an aggregate supply which depend only on prices and initial endowments.

The homotopy idea is that any economy is connected by an arc to some other economy that has a unique regular equilibrium. Along this arc, equilibrium moves in a continuous way without sliding off the boundary. It does not imply that at the end of the arc there is still a unique regular equilibrium. But, it implies that at the end of the arc the set of equilibria associated with the given economy is non-empty and compact. This is a kind of standard technique used in many contributions in general equilibrium theory, see for instance Villanacci and Zenginobuz (2005), del Mercato (2006), Mandel (2008), Bonnisseau and del Mercato (2008), Kung (2008), and Ericson and Kung (2015). However, the work of adapting this technique to economies with externalities on the production side is non-trivial and it requires some ingenious adjustments, because the production sets are not required to be convex with respect to the consumption and production activities of all the agents (for more details, see 
Subsection 4.2). Furthermore, our proof covers the case in which the economy is a standard private ownership economy without externalities. ${ }^{4}$

The technique described above is based on the homotopy invariance of the topological degree. Our proof is based on the topological degree modulo $2 .{ }^{5}$ The degree modulo 2 is simpler than the Brouwer degree which is based on the notion of oriented manifold. ${ }^{6}$ The reader can find in Geanakoplos and Shafer (1990) a brief review of both degree theories. In Section 6, we recall the definition and fundamental properties of the degree modulo 2.

We now compare our contribution with previous works. del Mercato (2006) and Balasko (2015) study pure exchange general equilibrium models with externalities. In Villanacci and Zenginobuz (2005), and Kung (2008), there are no externalities on the production side. In Ericson and Kung (2015), utility and transformation functions are also affected by the price system. In order to get their existence result, the authors need to perturb utility and transformation functions. ${ }^{7}$ Our contribution highlights that, in the presence of consumption and production externalities, there is no need for perturbing utility and transformation functions to establish the existence of an equilibrium. Furthermore, the contribution of Ericson and Kung (2015) presents some technical problems. ${ }^{8}$ The existence results of Arrow and Hahn (1971), Laffont and Laroque (1972), Bonnisseau and Médecin (2001), and Mandel (2008) are more general than ours. In Arrow and Hahn (1971), Laffont and Laroque (1972), and Bonnisseau and Médecin (2001), the authors use fixed point arguments, while we use differential techniques. The approach in Mandel (2008) differs from ours for two main reasons: the author uses the excess demand approach and the topological Brouwer degree. In order to use the excess demand approach, the author has to enlarge the commodity space treating the externalities as additional variables. Moreover, in Bonnisseau and Médecin (2001), and Mandel (2008), production technologies are also non-convex with respect to the individual firm's choice. For that reason, their equilibrium notion involves the concept of pricing rule and their existence proofs consist of techniques more sophisticated than those we use. In our simpler context, we provide an existence proof simpler than the ones provided in Bonnisseau and Médecin (2001), and Mandel (2008).

4 In Chapter 9 of Villanacci et al. (2002), the reader can find a homotopy proof for a standard private ownership economies without externalities. Our proof is simpler than the latter one, because we do not homotopize the shares.

5 See for instance, Chapter 4 in Milnor (1965), and Villanacci et al. (2002).

6 See for instance, Chapter 5 in Milnor (1965), and Mas-Colell (1985).

7 See pages 53-55 in Ericson and Kung (2015).

8 Some basic assumptions for the existence of an equilibrium are missing, i.e., the possibility of inaction and the compactness of the set of feasible allocations, or any related assumptions. See for instance, Assumption 1(2) and Assumption 3 in our paper, or Assumption P(2) and Assumption UB in Bonnisseau and Médecin (2001). 
The paper is organized as follows. In Section 2, we present the model and the assumptions. In Section 3, we provide the notions of competitive equilibrium and equilibrium function. The equilibrium function is built on the first-order conditions associated with households and firms maximization problems, and market clearing conditions. In Section 4, we first present our main result (Theorem 8) which states that the set of equilibria is non-empty and compact. Second, we provide the homotopy theorem (Theorem 9) used to prove Theorem 8. In order to apply Theorem 9, in Subsection 4.1 we build an appropriate private ownership economy that has a unique regular equilibrium. In Subsection 4.2 , we provide our homotopy and its properties. All the lemmas are proved in Section 5. Finally, in Section 6, the reader can find a brief review on the topological degree modulo 2.

\section{The model and the assumptions}

There is a finite number $C$ of physical commodities labeled by the superscript $c \in \mathcal{C}:=\{1, \ldots, C\}$. The commodity space is $\mathbb{R}^{C}$. There are a finite number $J$ of firms labeled by the subscript $j \in \mathcal{J}:=\{1, \ldots, J\}$ and a finite number $H$ of households labeled by the subscript $h \in \mathcal{H}:=\{1, \ldots, H\}$. Each firm is owned by households and it is characterized by a technology described by a transformation function. Each household is characterized by preferences described by a utility function, the shares on firms' profits and an endowment of commodities. Utility and transformation functions may be affected by the consumption and production activities of all other agents. The notations are summarized below.

- $y_{j}:=\left(y_{j}^{1}, . ., y_{j}^{c}, . ., y_{j}^{C}\right) \in \mathbb{R}^{C}$ is the production plan of firm $j$, as usual if $y_{j}^{c}>0$ then commodity $c$ is produced as an output, if $y_{j}^{\ell}<0$ then commodity $\ell$ is used as an input, $y_{-j}:=\left(y_{f}\right)_{f \neq j}$ denotes the production plan of firms other than $j, y:=\left(y_{j}\right)_{j \in \mathcal{J}}$.

- $x_{h}:=\left(x_{h}^{1}, \ldots, x_{h}^{c}, . ., x_{h}^{C}\right) \in \mathbb{R}_{++}^{C}$ denotes household $h$ 's consumption, $x_{-h}:=$ $\left(x_{k}\right)_{k \neq h}$ denotes the consumption of households other than $h, x:=\left(x_{h}\right)_{h \in \mathcal{H}}$.

- The production set of firm $j$ is described by a transformation function. This is a convenient way to represent a production set using an inequality on a function called the transformation function, see for instance Mas-Colell et al. (1995) and Villanacci et al. (2002). In the case of a single-output technology, the production set is commonly described by a production function. The transformation function is the counterpart of the production function in the case of production processes which involve several outputs.

The main innovation of this paper comes from the dependency of the transformation function $t_{j}$ on the production and consumption activities of all other agents. That is, $t_{j}$ describes both the technology of firm $j$ and the 
way in which firm $j$ 's technology is affected by the activities of the other agents. More precisely, for any given $\left(y_{-j}, x\right)$, the production set of the firm $j$ is given by the following set,

$$
Y_{j}\left(y_{-j}, x\right):=\left\{y_{j} \in \mathbb{R}^{C}: t_{j}\left(y_{j}, y_{-j}, x\right) \leq 0\right\}
$$

where the transformation function $t_{j}$ is a function from $\mathbb{R}^{C} \times \mathbb{R}^{C(J-1)} \times \mathbb{R}_{++}^{C H}$ to $\mathbb{R}, t:=\left(t_{j}\right)_{j \in \mathcal{J}}$.

- Household $h$ has preferences described by a utility function,

$$
u_{h}:\left(x_{h}, x_{-h}, y\right) \in \mathbb{R}_{++}^{C} \times \mathbb{R}_{+}^{C(H-1)} \times \mathbb{R}^{C J} \longrightarrow u_{h}\left(x_{h}, x_{-h}, y\right) \in \mathbb{R}
$$

$u_{h}\left(x_{h}, x_{-h}, y\right)$ is the utility level of household $h$ associated with $\left(x_{h}, x_{-h}, y\right)$. That is, $u_{h}$ also describes the way in which household $h$ 's preferences are affected by the actions of the other agents. Denote $u:=\left(u_{h}\right)_{h \in \mathcal{H}}$.

- $s_{j h} \in[0,1]$ is the share of firm $j$ owned by household $h ; s_{h}:=\left(s_{j h}\right)_{j \in \mathcal{J}} \in$ $[0,1]^{J}$ denotes the vector of the shares owned by household $h ; s:=\left(s_{h}\right)_{h \in \mathcal{H}} \in$ $[0,1]^{J H}$. As usual, $\sum_{h \in \mathcal{H}} s_{j h}=1$ for every firm $j \in \mathcal{J}$.

- $e_{h}:=\left(e_{h}^{1}, . ., e_{h}^{c}, . ., e_{h}^{C}\right) \in \mathbb{R}_{++}^{C}$ denotes household $h$ 's endowment, $e:=\left(e_{h}\right)_{h \in \mathcal{H}} \in$ $\mathbb{R}_{++}^{C H}$ and $r:=\sum_{h \in \mathcal{H}} e_{h} \in \mathbb{R}_{++}^{C}$.

- $E:=((u, e, s), t)$ is a private ownership economy with externalities.

- $p^{c}$ is the price of one unit of commodity $c, p:=\left(p^{1}, . ., p^{c}, \ldots, p^{C}\right) \in \mathbb{R}_{++}^{C}$.

- Given $w=\left(w^{1}, . ., w^{c}, . ., w^{C}\right) \in \mathbb{R}^{C}$, we denote $w^{\backslash}:=\left(w^{1}, . ., w^{c}, . ., w^{C-1}\right) \in$ $\mathbb{R}^{C-1}$

We make the following assumptions on the transformation functions.

Assumption 1 For all $j \in \mathcal{J}$,

(1) The function $t_{j}$ is continuous on its domain. For every $\left(y_{-j}, x\right) \in \mathbb{R}^{C(J-1)} \times$ $\mathbb{R}_{++}^{C H}$, the function $t_{j}\left(\cdot, y_{-j}, x\right)$ is differentiable and $D_{y_{j}} t_{j}(\cdot, \cdot, \cdot)$ is continuous on $\mathbb{R}^{C J} \times \mathbb{R}_{++}^{C H}$.

(2) For every $\left(y_{-j}, x\right) \in \mathbb{R}^{C(J-1)} \times \mathbb{R}_{++}^{C H}, t_{j}\left(0, y_{-j}, x\right)=0$.

(3) For every $\left(y_{-j}, x\right) \in \mathbb{R}^{C(J-1)} \times \mathbb{R}_{++}^{C H}$, the function $t_{j}\left(\cdot, y_{-j}, x\right)$ is differentiably strictly quasi-convex, i.e., it is a $C^{2}$ function and for all $y_{j}^{\prime} \in \mathbb{R}^{C}$, $D_{y_{j}}^{2} t_{j}\left(y_{j}^{\prime}, y_{-j}, x\right)$ is positive definite on $\operatorname{Ker} D_{y_{j}} t_{j}\left(y_{j}^{\prime}, y_{-j}, x\right) .{ }^{9}$

(4) For every $\left(y_{-j}, x\right) \in \mathbb{R}^{C(J-1)} \times \mathbb{R}_{++}^{C H}, D_{y_{j}} t_{j}\left(y_{j}^{\prime}, y_{-j}, x\right) \gg 0$ for all $y_{j}^{\prime} \in \mathbb{R}^{C}$.

9 Let $v$ and $v^{\prime}$ be two vectors in $\mathbb{R}^{n}, v \cdot v^{\prime}$ denotes the scalar product of $v$ and $v^{\prime}$. Let $A$ be a real matrix with $m$ rows and $n$ columns, and $B$ be a real matrix with $n$ rows and $l$ columns, $A B$ denotes the matrix product of $A$ and $B$. Without loss of generality, vectors are treated as row matrices and $A$ denotes both the matrix and the following linear mapping $A: v \in \mathbb{R}^{n} \rightarrow A(v):=A v^{T} \in \mathbb{R}^{[m]}$ where $v^{T}$ denotes the transpose of $v$ and $\mathbb{R}^{[m]}:=\left\{w^{T}: w \in \mathbb{R}^{m}\right\}$. When $m=1, A(v)$ coincides with the scalar product $A \cdot v$, treating $A$ and $v$ as vectors in $\mathbb{R}^{n}$. 
Fixing the externalities, the assumptions on $t_{j}$ are standard in a differentiable framework. From Points 1 and 4 of Assumption 1, the production set is a $C^{1}$ manifold with boundary of dimension $C$ and its boundary is a $C^{1}$ manifold of dimension $C-1$. Point 2 of Assumption 1 states that inaction is possible. Consequently, using standard arguments from profit maximization, the individual wealth of household $h$ derived from his endowment $e_{h} \in \mathbb{R}_{++}^{C}$ and his profit shares is strictly positive for every price $p \in \mathbb{R}_{++}^{C}$, from which one deduces the non-emptiness of the interior of the individual budget constraint. From Point 3 of Assumption 1, the production set is strictly convex. Furthermore, if the profit maximization problem has a solution then it is unique, because the function $t_{j}\left(\cdot, y_{-j}, x\right)$ is continuous and strictly quasi-convex. We remark that $t_{j}$ is not required to be quasi-convex with respect to all the variables, so we do not require the production set to be convex with respect to the externalities. From Point 4 of Assumption 1, the function $t_{j}\left(\cdot, y_{-j}, x\right)$ is strictly increasing and so the production set satisfies the classical "free disposal" property.

Remark 2 Our analysis holds true if some commodities are not involved in the technological process of firm $j$. In this case, for every firm $j$, one defines the set $\mathcal{C}_{j}$ of all the commodities $c \in \mathcal{C}$ that are involved in the technological process of firm $j$, where $C_{j}$ denotes the cardinality of the set $\mathcal{C}_{j}$ with $2 \leq C_{j} \leq C$. The production plan of firm $j$ is then defined as $y_{j}:=\left(y_{j}^{c}\right)_{c \in \mathcal{C}_{j}} \in \mathbb{R}^{C_{j}}$ and the transformation function $t_{j}$ is a function from $\mathbb{R}^{C_{j}} \times \prod_{f \neq j} \mathbb{R}^{C_{f}} \times \mathbb{R}_{++}^{C H}$ to $\mathbb{R}$. In this case, all the assumptions on the transformation functions are written just replacing $\mathbb{R}^{C} \times \mathbb{R}^{C(J-1)} \times \mathbb{R}_{++}^{C H}$ by $\mathbb{R}^{C_{j}} \times \prod_{f \neq j} \mathbb{R}^{C_{f}} \times \mathbb{R}_{++}^{C H}$. Furthermore, in the definition of a competitive equilibrium, one also adapts the market clearing condition for every commodity $c$ by considering only the firms that use commodity $c$ in their technological process. That is, for every commodity $c$, the sum over $j \in \mathcal{J}$ is replaced by the sum over $j \in \mathcal{J}(c):=\left\{j \in \mathcal{J}: c \in \mathcal{C}_{j}\right\}$.

For any given externality $(x, y) \in \mathbb{R}_{++}^{C H} \times \mathbb{R}^{C J}$, define the set of all the production allocations that are consistent with the externality $(x, y)$, that is

$$
Y(x, y):=\left\{y^{\prime} \in \mathbb{R}^{C J}: t_{j}\left(y_{j}^{\prime}, y_{-j}, x\right) \leq 0, \forall j \in \mathcal{J}\right\}
$$

We remind that $r=\sum_{h \in \mathcal{H}} e_{h}$ and we make the following assumption which is analogous to Assumption UB (Uniform Boundedness) in Bonnisseau and Médecin (2001), and Assumption P(3) in Mandel (2008).

Assumption 3 (Uniform Boundedness) There exists a bounded set $C(r) \subseteq$ $\mathbb{R}^{C J}$ such that for every $(x, y) \in \mathbb{R}_{++}^{C H} \times \mathbb{R}^{C J}$,

$$
Y(x, y) \cap\left\{y^{\prime} \in \mathbb{R}^{C J}: \sum_{j \in \mathcal{J}} y_{j}^{\prime}+r \gg 0\right\} \subseteq C(r)
$$


The following lemma is an immediate consequence of Assumption 3.

Lemma 4 There exists a bounded set $K(r) \subseteq \mathbb{R}_{++}^{C H} \times \mathbb{R}^{C J}$ such that for every $(x, y) \in \mathbb{R}_{++}^{C H} \times \mathbb{R}^{C J}$, the following set is included in $K(r)$.

$$
A(x, y ; r):=\left\{\left(x^{\prime}, y^{\prime}\right) \in \mathbb{R}_{++}^{C H} \times \mathbb{R}^{C J}: y^{\prime} \in Y(x, y) \text { and } \sum_{h \in \mathcal{H}} x_{h}^{\prime}-\sum_{j \in \mathcal{J}} y_{j}^{\prime} \leq r\right\}
$$

It is well known that the boundedness of the set of feasible allocations is a crucial condition for the non-emptiness and the compactness of the set of equilibria. Fixing the externalities, from Assumption 3 one deduces that the set of feasible allocations is bounded. Therefore, in this sense, Assumption 3 is standard. Furthermore, Assumption 3 also guarantees that the set of feasible allocations $A(x, y ; r)$ is uniformly bounded with respect to the externalities $(x, y)$. In particular, it implies that the set $\mathcal{F}(r)=\left\{(x, y) \in \mathbb{R}_{++}^{C H} \times \mathbb{R}^{C J} \mid t_{j}\left(y_{j}, y_{-j}, x\right) \leq\right.$ $0, \forall j \in \mathcal{J}$ and $\left.\sum_{h \in \mathcal{H}} x_{h}-\sum_{j \in \mathcal{J}} y_{j} \leq r\right\}$ of feasible allocations that are mutually consistent is bounded. However, for a proof of the existence of an equilibrium it would not be sufficient to assume only the boundedness of the set $\mathcal{F}(r) .{ }^{10} \mathrm{We}$ use Lemma 4 to prove that the homotopy is compact once externalities move along the homotopy arc, see Step 2.1 in the proof of Proposition 15 in Section 5 .

We make the following assumptions on the utility functions.

Assumption 5 For all $h \in \mathcal{H}$

(1) The function $u_{h}$ is continuous on its domain. For every $\left(x_{-h}, y\right) \in \mathbb{R}_{++}^{C(H-1)} \times$ $\mathbb{R}^{C J}$, the function $u_{h}\left(\cdot, x_{-h}, y\right)$ is differentiable and $D_{x_{h}} u_{h}(\cdot, \cdot, \cdot)$ is continuous on $\mathbb{R}_{++}^{C H} \times \mathbb{R}^{C J}$.

(2) For every $\left(x_{-h}, y\right) \in \mathbb{R}_{++}^{C(H-1)} \times \mathbb{R}^{C J}$, the function $u_{h}\left(\cdot, x_{-h}, y\right)$ is differentiably strictly increasing, i.e., $D_{x_{h}} u_{h}\left(x_{h}^{\prime}, x_{-h}, y\right) \gg 0$ for all $x_{h}^{\prime} \in \mathbb{R}_{++}^{C}$.

(3) For every $\left(x_{-h}, y\right) \in \mathbb{R}_{++}^{C(H-1)} \times \mathbb{R}^{C J}$, the function $u_{h}\left(\cdot, x_{-h}, y\right)$ it is differentiably strictly quasi-concave, i.e., it is $C^{2}$ and for all $x_{h}^{\prime} \in \mathbb{R}_{++}^{C}$, $D_{x_{h}}^{2} u_{h}\left(x_{h}^{\prime}, x_{-h}, y\right)$ is negative definite on $\operatorname{Ker} D_{x_{h}} u_{h}\left(x_{h}^{\prime}, x_{-h}, y\right)$.

(4) For every $\left(x_{-h}, y\right) \in \mathbb{R}_{+}^{C(H-1)} \times \mathbb{R}^{C J}$ and for every $u \in \operatorname{Im} u_{h}\left(\cdot, x_{-h}, y\right)$,

$$
\mathrm{cl}_{\mathbb{R}^{C}}\left\{x_{h} \in \mathbb{R}_{++}^{C}: u_{h}\left(x_{h}, x_{-h}, y\right) \geq u\right\} \subseteq \mathbb{R}_{++}^{C}
$$

Fixing the externalities, the assumptions on $u_{h}$ are standard in a differentiable framework. From Point 3 of Assumption 5, the upper contour sets are strictly convex. Consequently, if the utility maximization problem has a solution then

${ }^{10}$ See Section 2 in Chapter 6 of Arrow and Hahn (1971), Bonnisseau and Médecin (2001), and Mandel (2008), where these authors also need uniform boundedness assumptions in order to prove the non-emptiness of the set of equilibria. 
it is unique. Notice that $u_{h}$ is not required to be quasi-concave with respect to all the variables, that is, we do not require preferences to be convex with respect to the externalities. Point 4 of Assumption 5 is the classical Boundary Condition (BC), it means that the closure of the upper counter sets is included in $\mathbb{R}_{++}^{C}$. We notice that in Point 1 and Point 4 of Assumption 5, we allow for consumption externalities $x_{-h}$ on the boundary of the set $\mathbb{R}_{++}^{C(H-1)}$ in order to look at the limit of the behavior of $u_{h}$ with respect to the consumption externalities. It means that $\mathrm{BC}$ is still valid whenever consumption externalities converge to zero for some commodities. This property is used in Step 2.2 of the proof of Proposition 15 in Section $5 .{ }^{11}$ If $u_{h}$ does not satisfy BC whenever consumption externalities converge to zero for some commodities, we provide below an alternative assumption on $u_{h}$ from which one still gets Step 2.2 in the proof of Proposition 15, while maintaining Point 1 and Point 4 of Assumption 5 only for consumption externalities in $\mathbb{R}_{++}^{C(H-1)} \cdot{ }^{12}$

(5) There exists $\delta>0$ such that for every $\left(x_{-h}, y\right) \in \mathbb{R}_{++}^{C(H-1)} \times \mathbb{R}^{C J}$ and for every $\left(x_{h}, x_{h}^{\prime}\right) \in \mathbb{R}_{++}^{2 C}$, if $u_{h}\left(x_{h}, x_{-h}, y\right)>u_{h}\left(x_{h}^{\prime}, x_{-h}, y\right)$, then $u_{h}\left(x_{h}, x_{-h}+\right.$ $\delta \mathbf{1}, y) \geq u_{h}\left(x_{h}^{\prime}, x_{-h}+\delta \mathbf{1}, y\right)$ where $\mathbf{1}:=(1, \ldots, 1) \in \mathbb{R}_{++}^{C} \cdot{ }^{13}$

\section{Competitive equilibrium and equilibrium function}

In this section, we provide the definition of competitive equilibrium à la Nash and the notion of equilibrium function.

Without loss of generality, commodity $C$ is the "numeraire good". Then, given $p \backslash \in \mathbb{R}_{++}^{C-1}$ with innocuous abuse of notation, we denote $p:=(p \backslash, 1) \in \mathbb{R}_{++}^{C}$.

Definition 6 (Competitive equilibrium) $\left(x^{*}, y^{*}, p^{*}\right) \in \mathbb{R}_{++}^{C H} \times \mathbb{R}^{C J} \times \mathbb{R}_{++}^{C-1}$ is a competitive equilibrium for the economy $E$ if for all $j \in \mathcal{J}$, $y_{j}^{*}$ solves the following problem

$$
\begin{aligned}
& \max _{y_{j} \in \mathbb{R}^{C}} p^{*} \cdot y_{j} \\
& \text { subject to } t_{j}\left(y_{j}, y_{-j}^{*}, x^{*}\right) \leq 0
\end{aligned}
$$

\footnotetext{
${ }^{11}$ A simple example of utility function that satisfies this property is given by any additively separable function $u_{h}\left(x_{h}, x_{-h}\right)=\widetilde{u}_{h}\left(x_{h}\right)+v_{h}\left(x_{-h}\right)$ where $\widetilde{u}_{h}$ is defined on $\mathbb{R}_{++}^{C}$ and satisfies the classical BC, and $v_{h}$ is defined on $\mathbb{R}_{+}^{C(H-1)}$.

${ }^{12}$ For example, consider two commodities and the utility function $u_{h}\left(x_{h}^{1}, x_{h}^{2}, x_{k}^{1}\right):=$ $x_{h}^{1} x_{h}^{2} x_{k}^{1}$ where $\left(x_{h}^{1}, x_{h}^{2}\right) \in \mathbb{R}_{++}^{2}$ and $x_{k}^{1} \in \mathbb{R}_{+}$. This function does not satisfy BC whenever the externality $x_{k}^{1}$ converges to zero, but it satisfies Point 5 .

${ }^{13}$ A simple example of utility function that satisfies Point 5 is given by any multiplicatively separable function $u_{h}\left(x_{h}, x_{-h}\right)=\widetilde{u}_{h}\left(x_{h}\right) m_{h}\left(x_{-h}\right)$ where $\widetilde{u}_{h}$ is defined on $\mathbb{R}_{++}^{C}$ and $m_{h}\left(x_{-h}\right)>0$ for every $x_{-h} \in \mathbb{R}_{++}^{C(H-1)}$.
} 
for all $h \in \mathcal{H}, x_{h}^{*}$ solves the following problem

$$
\begin{aligned}
& \max _{x_{h} \in \mathbb{R}_{++}^{C}} u_{h}\left(x_{h}, x_{-h}^{*}, y^{*}\right) \\
& \text { subject to } p^{*} \cdot x_{h} \leq p^{*} \cdot\left(e_{h}+\sum_{j \in \mathcal{J}} s_{j h} y_{j}^{*}\right)
\end{aligned}
$$

and $\left(x^{*}, y^{*}\right)$ satisfies market clearing conditions, that is

$$
\sum_{h \in \mathcal{H}} x_{h}^{*}=\sum_{h \in \mathcal{H}} e_{h}+\sum_{j \in \mathcal{J}} y_{j}^{*}
$$

Using first-order conditions, one easily characterizes the solutions of firms and households maximization problems. The proof of the following proposition is standard, because in problems (2) and (3) each agent takes as given both the price system and the choices of the other agents.

\section{Proposition 7}

(1) From Assumption 1, if $y_{j}^{*}$ is a solution to problem (2), then it is unique and it is completely characterized by KKT conditions. ${ }^{14}$

(2) From Point 2 of Assumption 1 and Assumption 5, there exists a unique solution $x_{h}^{*}$ to problem (3) and it is completely characterized by KKT conditions.

(3) As usual, from Point 2 of Assumption 5, household h's budget constraint holds with an equality. Thus, at equilibrium, due to the Walras law, the market clearing condition for commodity $C$ is "redundant". Then, one replaces condition (4) with $\sum_{h \in \mathcal{H}} x_{h}^{* \backslash}=\sum_{h \in \mathcal{H}} e_{h}^{\backslash}+\sum_{j \in \mathcal{J}} y_{j}^{* \backslash}$.

Let $\Xi:=\left(\mathbb{R}_{++}^{C} \times \mathbb{R}_{++}\right)^{H} \times\left(\mathbb{R}^{C} \times \mathbb{R}_{++}\right)^{J} \times \mathbb{R}_{++}^{C-1}$ be the set of endogenous variables with generic element $\xi:=(x, \lambda, y, \alpha, p \backslash):=\left(\left(x_{h}, \lambda_{h}\right)_{h \in \mathcal{H}},\left(y_{j}, \alpha_{j}\right)_{j \in \mathcal{J}}, p \backslash\right)$ where $\lambda_{h}$ denotes the Lagrange multiplier associated with household $h$ 's budget constraint, and $\alpha_{j}$ denotes the Lagrange multiplier associated with firm $j$ 's technological constraint. We can now describe the competitive equilibria associated with the economy $E$ using the equilibrium function $F: \Xi \rightarrow \mathbb{R}^{\operatorname{dim} \Xi}$,

$$
F(\xi):=\left(\left(F^{h .1}(\xi), F^{h .2}(\xi)\right)_{h \in \mathcal{H}},\left(F^{j .1}(\xi), F^{j .2}(\xi)\right)_{j \in \mathcal{J}}, F^{M}(\xi)\right)
$$

where $F^{h .1}(\xi):=D_{x_{h}} u_{h}\left(x_{h}, x_{-h}, y\right)-\lambda_{h} p, F^{h .2}(\xi):=-p \cdot\left(x_{h}-e_{h}-\sum_{j \in \mathcal{J}} s_{j h} y_{j}\right)$, $F^{j .1}(\xi):=p-\alpha_{j} D_{y_{j}} t_{j}\left(y_{j}, y_{-j}, x\right), F^{j .2}(\xi):=-t_{j}\left(y_{j}, y_{-j}, x\right)$, and $F^{M}(\xi):=$ $\sum_{h \in \mathcal{H}} x_{h}^{\backslash}-\sum_{j \in \mathcal{J}} y_{j}^{\backslash}-\sum_{h \in \mathcal{H}} e_{h}^{\curlywedge}$

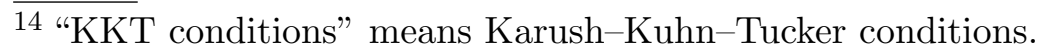


$\xi^{*}=\left(x^{*}, \lambda^{*}, y^{*}, \alpha^{*}, p^{*}\right) \in \Xi$ is an extended equilibrium for the economy $E$ if and only if $F\left(\xi^{*}\right)=0$. By Proposition $7,\left(x^{*}, y^{*}, p^{*}\right)$ is a competitive equilibrium for $E$ if and only if there exists $\left(\lambda^{*}, \alpha^{*}\right)$ such that $\xi^{*}$ is an extended equilibrium for $E$. We simply call $\xi^{*}$ an equilibrium.

\section{Existence and compactness}

In this section, we show that the set of competitive equilibria with strictly positive consumptions and strictly positive prices is compact and non-empty. The result is provided in the following theorem.

Theorem 8 The equilibrium set $F^{-1}(0)$ is compact and non-empty.

In order to prove Theorem 8, we use a homotopy approach following the seminal paper by Smale (1974). The following theorem is a consequence of the homotopy invariance of the topological degree. Following Chapter 4 in Milnor (1965), and more recent contributions by Villanacci and Zenginobuz (2005), del Mercato (2006) and Bonnisseau and del Mercato (2008), our homotopy approach is based on the theory of degree modulo 2. The reader can find a survey of the degree theory, for example, in Geanakoplos and Shafer (1990) and in Villanacci et al. (2002). In Section 6, we recall the definition and fundamental properties of the degree modulo 2 .

Theorem 9 (Homotopy Theorem) Let $M$ and $N$ be $C^{2}$ manifolds of the same dimension contained in euclidean spaces. Let $y \in N$ and $f, g: M \rightarrow N$ be two functions such that $f$ is continuous, $g$ is $C^{1}$, $y$ is a regular value of $g$ and $\# g^{-1}(y)$ is odd. Let $L$ be a continuous homotopy from $g$ to $f$ such that $L^{-1}(y)$ is compact. Then,

(1) $g^{-1}(y)$ is compact and $\operatorname{deg}_{2}(g, y)=1$,

(2) $f^{-1}(y)$ is compact and $\operatorname{deg}_{2}(f, y)=1$.

The equilibrium function $F$ plays the role of function $f$ given in Theorem 9 . In order to construct the function playing the role of function $g$, we proceed as follows. In Subsection 4.1, we construct an appropriate economy called the "test economy". $G$ is the equilibrium function associated with the test economy and it plays the role of function $g$ in Theorem 9. The test economy has an unique regular equilibrium, i.e., $\# G^{-1}(0)=1$ and 0 is a regular value of $G$, see Proposition 12. In Subsection 4.2, we provide the homotopy $H$ from $G$ to $F$ playing the role of the homotopy $L$. Proposition 15 states the compactness of $H^{-1}(0)$. Using Propositions 12 and 15, all the assumptions of Theorem 9 are satisfied, and then one gets the following lemma. 
Lemma $10 F^{-1}(0)$ is compact and $\operatorname{deg}_{2}(F, 0)=1$.

Using Lemma 10 and the non-triviality property of the topological degree, one gets $F^{-1}(0) \neq \emptyset$, and then Theorem 8 is completely proved.

Finally, we remark that if, in addition, $E$ is a regular economy (i.e., the equilibrium function $F$ is $C^{1}$ and 0 is a regular value of $F$ ), then using Lemma 10 and the computation of the degree modulo 2 , one obviously obtains that, at a regular economy, the number of equilibria is finite and odd. ${ }^{15}$ However, this paper does not address any regularity issue. In the presence of consumption and production externalities, the analysis of regular economies is quite sensitive. For the case without externalities on the production side, see for example Bonnisseau (2003), Kung (2008), and Bonnisseau and del Mercato (2010). In the presence of production externalities, the analysis of regular economies deserves a separate analysis, see del Mercato and Platino (forthcoming 2017).

\subsection{The "test economy" and its properties}

We construct the test economy in two steps. We first fix the externalities and we consider a Pareto optimal allocation of a standard production economy $\bar{E}$ without externalities. Second, using the Second Theorem of Welfare Economics, we construct an appropriate private ownership economy $\widetilde{E}$ that has a unique regular equilibrium. $\widetilde{E}$ is called the "test economy" and it is an economy without externalities.

Fix an allocation $(\bar{x}, \bar{y}) \in \mathbb{R}_{++}^{C H} \times \mathbb{R}^{C J}$. Define $\bar{u}_{h}\left(x_{h}\right):=u_{h}\left(x_{h}, \bar{x}_{-h}, \bar{y}\right)$ for all $h \in \mathcal{H}, \bar{t}_{j}\left(y_{j}\right):=t_{j}\left(y_{j}, \bar{y}_{-j}, \bar{x}\right)$ for all $j \in \mathcal{J}$, and the production economy $\bar{E}:=$ $(\bar{u}, \bar{t}, r)$ where $r=\sum_{h \in \mathcal{H}} e_{h}$. Under Assumptions 1, 3 and 5, there exist a Pareto optimal allocation $(\widetilde{x}, \widetilde{y})$ of the economy $\bar{E}$ and Lagrange multipliers $(\widetilde{\theta}, \widetilde{\gamma}, \widetilde{\beta})$ such that $(\widetilde{x}, \widetilde{y}, \widetilde{\theta}, \widetilde{\gamma}, \widetilde{\beta})$ is completely characterized by the first-order conditions for Pareto optimality. This result is provided in the following proposition.

Proposition 11 There exist a Pareto optimal allocation $(\widetilde{x}, \widetilde{y}) \in \mathbb{R}_{++}^{C H} \times \mathbb{R}^{C J}$ of the economy $\bar{E}$ and $(\widetilde{\beta}, \widetilde{\theta}, \widetilde{\gamma})=\left(\left(\widetilde{\beta}_{j}\right)_{j \in \mathcal{J}},\left(\widetilde{\theta}_{h}\right)_{h \neq 1}, \widetilde{\gamma}\right) \in \mathbb{R}_{++}^{J} \times \mathbb{R}_{++}^{H-1} \times \mathbb{R}_{++}^{C}$ such that $(\widetilde{x}, \widetilde{y}, \widetilde{\beta}, \widetilde{\theta}, \widetilde{\gamma})$ is the unique solution to the following system.

$\left\{\begin{array}{l}(1) D_{x_{1}} \bar{u}_{1}\left(x_{1}\right)=\gamma(2) \forall h \neq 1, \theta_{h} D_{x_{h}} \bar{u}_{h}\left(x_{h}\right)=\gamma(3) \forall h \neq 1, \bar{u}_{h}\left(x_{h}\right)=\bar{u}_{h}\left(\widetilde{x}_{h}\right) \\ (4) \forall j \in \mathcal{J}, \gamma=\beta_{j} D_{y_{j}} \bar{t}_{j}\left(y_{j}\right)(5) \forall j \in \mathcal{J},-\bar{t}_{j}\left(y_{j}\right)=0 \text { (6) } \sum_{h \in \mathcal{H}} x_{h}-\sum_{j \in \mathcal{J}} y_{j}=r\end{array}\right.$

$\overline{15}$ The computation of the degree modulo 2 for $C^{1}$ functions and regular values is given by Proposition 17 in Section 6 . 
It is well known that a Pareto optimal allocation can be supported by some price system. ${ }^{16}$ From system (6), one easily deduces a supporting price $\widetilde{p}$, a redistribution of initial endowments $\widetilde{e}=\left(\widetilde{e}_{h}\right)_{h \in \mathcal{H}}$ and the equilibrium equations satisfied by $(\widetilde{x}, \widetilde{y})$ for appropriate Lagrange multipliers $(\widetilde{\lambda}, \widetilde{\alpha}) \in \mathbb{R}_{++}^{H} \times \mathbb{R}_{++}^{J}$. More precisely, first define

$$
\widetilde{e}_{h}:=\widetilde{x}_{h}-\sum_{j \in \mathcal{J}} s_{j h} \widetilde{y}_{j}
$$

and the economy $\widetilde{E}:=((\bar{u}, \widetilde{e}, s), \bar{t})$ which is a private ownership economy

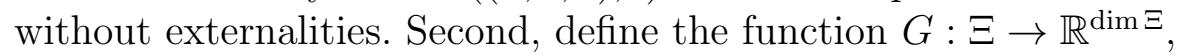

$$
G(\xi):=\left(\left(G^{h .1}(\xi), G^{h .2}(\xi)\right)_{h \in \mathcal{H}},\left(G^{j .1}(\xi), G^{j .2}(\xi)\right)_{j \in \mathcal{J}}, G^{M}(\xi)\right)
$$

where $G^{h .1}(\xi):=D_{x_{h}} u_{h}\left(x_{h}, \bar{x}_{-h}, \bar{y}\right)-\lambda_{h} p, G^{h .2}(\xi):=-p \cdot\left(x_{h}-\widetilde{e}_{h}-\sum_{j \in \mathcal{J}} s_{j h} y_{j}\right)$, $G^{j .1}(\xi):=p-\alpha_{j} D_{y_{j}} t_{j}\left(y_{j}, \bar{y}_{-j}, \bar{x}\right), G^{j .2}(\xi):=-t_{j}\left(y_{j}, \bar{y}_{-j}, \bar{x}\right)$ and $G^{M}(\xi):=$ $\sum_{h \in \mathcal{H}} x_{h}^{\backslash}-\sum_{j \in \mathcal{J}} y_{j}^{\backslash}-\sum_{h \in \mathcal{H}} \widetilde{e}_{h}$.

The function $G$ is nothing else than the equilibrium function associated with the economy $\widetilde{E}$. Finally, define $\widetilde{\xi}:=\left(\widetilde{x}, \widetilde{\lambda}, \widetilde{y}, \widetilde{\alpha}, \widetilde{p}^{\backslash}\right)$ with $\widetilde{p}:=\frac{\widetilde{\gamma}}{\widetilde{\gamma}^{C}}, \widetilde{\lambda}_{1}:=\widetilde{\gamma}^{C}$, $\widetilde{\lambda}_{h}:=\frac{\widetilde{\gamma}^{C}}{\widetilde{\theta}_{h}}$ for all $h \neq 1$ and $\widetilde{\alpha}_{j}:=\frac{\widetilde{\beta}_{j}}{\widetilde{\gamma}^{C}}$ for all $j \in \mathcal{J}$. Using system (6), it is an easy matter to check that $G(\widetilde{\xi})=0$. As stated in the following proposition, $\widetilde{\xi}$ is the unique regular equilibrium of the economy $\widetilde{E}$.

Proposition $12 G^{-1}(0)=\{\widetilde{\xi}\}, G$ is $C^{1}$ and 0 is a regular value for $G$.

Remark 13 Notice that the redistribution of endowments $\widetilde{e}=\left(\widetilde{e}_{h}\right)_{h \in \mathcal{H}}$ given in (7) is not necessarily strictly positive. However, at equilibrium, the individual wealth of household $h$ is equal to $\widetilde{p} \cdot \widetilde{x}_{h}$ which is strictly positive. One might wish to consider a different redistribution that gives rise to positive endowments. But, such a redistribution involves also some redistributions of the shares. ${ }^{17}$ Our redistribution of endowments $\widetilde{e}=\left(\widetilde{e}_{h}\right)_{h \in \mathcal{H}}$ does not involve any redistribution of shares, so that we do not need to homotopize the shares, see the homotopy $H$ in the next subsection. ${ }^{18}$

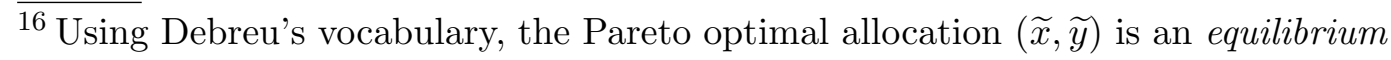
relative to some price system, see Section 6.4 in Debreu (1959).

${ }^{17}$ For example, the redistribution $\widehat{e}_{h}:=\widehat{s}_{j h} \sum_{h \in \mathcal{H}} e_{h}$ with $\widehat{s}_{j h}:=\frac{\widetilde{p} \cdot \widetilde{x}_{h}}{\widetilde{p} \cdot \sum_{h \in \mathcal{H}} \widetilde{x}_{h}}$.

${ }^{18}$ Even in the absence of externalities, homotopizing the shares complicates the homotopy proof, see for instance Section 2.2 in Chapter 9 of Villanacci et al. (2002). 


\subsection{The homotopy and its properties}

The basic idea is to homotopize the endowments and the externalities by a segment in the two economies $\widetilde{E}$ and $E$. But, one finds the following difficulty. At equilibrium, the individual wealth is positive at the beginning as well as at the end of the homotopy arc. ${ }^{19}$ Nevertheless, since the production sets are not required to be convex with respect to the choices of all agents, the equilibrium individual wealth may not be positive along the homotopy arc. Consequently, the individual budget constraints may be empty along the homotopy arc. We illustrate the details below.

First, homotopize the endowments by a segment. Then, for every $\tau \in[0,1]$ the individual wealth is given by $p \cdot\left[\tau e_{h}+(1-\tau) \widetilde{e}_{h}\right]+p \cdot \sum_{j \in \mathcal{J}} s_{j h} y_{j}$ which is equal to

$$
p \cdot\left[\tau e_{h}+(1-\tau) \widetilde{x}_{h}\right]+p \cdot \sum_{j \in \mathcal{J}} s_{j h}\left[y_{j}-(1-\tau) \widetilde{y}_{j}\right]
$$

Thus, the individual wealth is positive if $p \cdot y_{j} \geq p \cdot(1-\tau) \widetilde{y}_{j}$ for every $j \in$ $\mathcal{J}$. Using standard arguments from profit maximization, at equilibrium, this condition is satisfied if $(1-\tau) \widetilde{y}_{j}$ belongs to the production set of firm $j$. On the other hand if, at the same time, one homotopizes the externalities by a segment, the individual production set becomes the following set,

$$
Y_{j}\left(\tau y_{-j}+(1-\tau) \bar{y}_{-j}, \tau x+(1-\tau) \bar{x}\right)
$$

But, one does not know whether or not the production plan $(1-\tau) \widetilde{y}_{j}$ belongs to the production set given above, unless the transformation function $t_{j}$ is quasiconvex with respect to all the variables. Indeed, $t_{j}\left(0, y_{-j}, x\right)=0$ because of the possibility of inaction, and $t_{j}\left(\widetilde{y}_{j}, \bar{y}_{-j}, \bar{x}\right)=0$ because $G(\widetilde{\xi})=0$. If $t_{j}$ is quasi-convex with respect to all the variables, then $t_{j}\left((1-\tau) \widetilde{y}_{j}, \tau y_{-j}+(1-\right.$ $\left.\tau) \bar{y}_{-j}, \tau x+(1-\tau) \bar{x}\right) \leq 0$, and so $(1-\tau) \widetilde{y}_{j}$ belongs to the production set given above. But, $t_{j}$ is not required to be quasi-convex with respect to all the variables.

In order to overcome the difficulty described above, we define below the homotopy $H$ from the function $G$ to the equilibrium function $F$ in two times, by using the two homotopies $\Phi$ and $\Gamma$ defined below. Namely,

- in the first homotopy $\Phi$, we homotopize the endowments by a segment, without homotopizing the externalities,

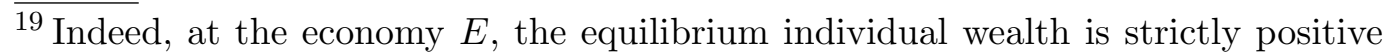
because of the possibility of inaction (Point 2 of Assumption 1) and standard arguments from profit maximization.
} 
- in the second homotopy $\Gamma$, we homotopize the externalities by a segment in utility and transformation functions, without homotopizing the endowments.

Remark 14 Notice that,

(1) If the externalities are fixed, then only one homotopy is needed, namely the homotopy $\Phi$. Then, our homotopy proof covers the case in which the economy $E$ is a standard private ownership economy without externalities.

(2) If the production sets are convex with respect to the production and consumption activities of all agents, then endowments and externalities can be homotopized at the same time.

We now provide the definition of all our homotopies. First, define the following convex combinations,

$$
e_{h}(\tau):=\tau e_{h}+(1-\tau) \widetilde{e}_{h}, x(\tau):=\tau x+(1-\tau) \bar{x}, y(\tau):=\tau y+(1-\tau) \bar{y}
$$

and the two following homotopies $\Phi, \Gamma: \Xi \times[0,1] \rightarrow \mathbb{R}^{\operatorname{dim} \Xi}$,

$$
\Phi(\xi, \tau):=\left(\left(\Phi^{h .1}(\xi, \tau), \Phi^{h .2}(\xi, \tau)\right)_{h \in \mathcal{H}},\left(\Phi^{j .1}(\xi, \tau), \Phi^{j .2}(\xi, \tau)\right)_{j \in \mathcal{J}}, \Phi^{M}(\xi, \tau)\right)
$$

where $\Phi^{h .1}(\xi, \tau)=D_{x_{h}} u_{h}\left(x_{h}, \bar{y}_{-j}, \bar{x}\right)-\lambda_{h} p, \Phi^{h .2}(\xi, \tau)=-p \cdot\left[x_{h}-e_{h}(\tau)-\right.$ $\left.\sum_{j \in \mathcal{J}} s_{j h} y_{j}\right], \Phi^{j .1}(\xi, \tau)=p-\alpha_{j} D_{y_{j}} t_{j}\left(y_{j}, \bar{y}_{-j}, \bar{x}\right), \Phi^{j .2}(\xi, \tau)=-t_{j}\left(y_{j}, \bar{y}_{-j}, \bar{x}\right)$, $\left.\Phi^{M}(\xi, \tau)=\sum_{h \in \mathcal{H}} x_{h}^{\backslash}-\sum_{j \in \mathcal{J}} y_{j}-\sum_{h \in \mathcal{H}} e_{h}(\tau)\right\rfloor$.

$$
\Gamma(\xi, \tau):=\left(\left(\Gamma^{h .1}(\xi, \tau), \Gamma^{h .2}(\xi, \tau)\right)_{h \in \mathcal{H}},\left(\Gamma^{j .2}(\xi, \tau), \Gamma^{j .2}(\xi, \tau)\right)_{j \in \mathcal{J}}, \Gamma^{M}(\xi, \tau)\right)
$$

where $\Gamma^{h .1}(\xi, \tau)=D_{x_{h}} u_{h}\left(x_{h}, x_{-h}(\tau), y(\tau)\right)-\lambda_{h} p, \Gamma^{h .2}(\xi, \tau)=-p \cdot\left[x_{h}-e_{h}-\right.$ $\left.\sum_{j \in \mathcal{J}} s_{j h} y_{j}\right], \Gamma^{j .1}(\xi, \tau)=p-\alpha_{j} D_{y_{j}} t_{j}\left(y_{j}, y_{-j}(\tau), x(\tau)\right), \Gamma^{j .2}(\xi, \tau)=-t_{j}\left(y_{j}, y_{-j}(\tau), x(\tau)\right)$, $\Gamma^{M}(\xi, \tau)=\sum_{h \in \mathcal{H}} x_{h}^{\backslash}-\sum_{j \in \mathcal{J}} y_{j}^{\backslash}-\sum_{h \in \mathcal{H}} e_{h}^{\backslash}$

Define then the homotopy $H: \Xi \times[0,1] \rightarrow \mathbb{R}^{\operatorname{dim} \Xi}$,

$$
H(\xi, \psi):=\left\{\begin{array}{l}
\Phi(\xi, 2 \psi) \text { if } 0 \leq \psi \leq \frac{1}{2} \\
\Gamma(\xi, 2 \psi-1) \text { if } \frac{1}{2} \leq \psi \leq 1
\end{array}\right.
$$

The homotopy $H$ is continuous since $\Phi$ and $\Gamma$ are composed by continuous functions. Importantly, $H\left(\xi, \frac{1}{2}\right)$ is well defined since $\Phi(\xi, 1)=\Gamma(\xi, 0)$. Furthermore, $H(\xi, 0)=\Phi(\xi, 0)=G(\xi)$ and $H(\xi, 1)=\Gamma(\xi, 1)=F(\xi)$. We conclude the section by stating the following proposition. 
Proposition $15 H^{-1}(0)$ is compact.

\section{Proofs}

Proof of Lemma 4. Let $\left(x^{\prime}, y^{\prime}\right) \in A(x, y ; r)$. Since $\sum_{h \in \mathcal{H}} x_{h}^{\prime} \gg 0, y^{\prime}$ belongs to the bounded set $C(r)$ given by Assumption 3. Furthermore, for every $h \in \mathcal{H}$, $0 \ll x_{h}^{\prime} \ll \sum_{h \in \mathcal{H}} x_{h}^{\prime} \leq \sum_{j \in \mathcal{J}} y_{j}^{\prime}+r$. Thus, there exists a bounded set $K(r) \subseteq$ $\mathbb{R}_{++}^{C H} \times \mathbb{R}^{C J}$ such that for every $(x, y) \in \mathbb{R}_{++}^{C H} \times \mathbb{R}^{C J}, A(x, y ; r) \subseteq K(r)$.

Proof of Proposition 11. Let $\bar{E}$ be the production economy defined in Section 4.1. We remind that $A(\bar{x}, \bar{y} ; r):=\left\{\left(x^{\prime}, y^{\prime}\right) \in \mathbb{R}_{++}^{C H} \times \mathbb{R}^{C J}: \bar{t}_{j}\left(y_{j}^{\prime}\right) \leq\right.$ $0, \forall j \in \mathcal{J}$ and $\left.\sum_{h \in \mathcal{H}} x_{h}^{\prime}-\sum_{j \in \mathcal{J}} y_{j}^{\prime} \leq r\right\}$. Consider the set $U(r):=\left\{\left(u_{h}^{\prime}\right)_{h \in \mathcal{H}} \in\right.$ $\left.\prod_{h \in \mathcal{H}} \operatorname{Im} \bar{u}_{h} \mid \exists\left(x^{\prime}, y^{\prime}\right) \in A(\bar{x}, \bar{y} ; r): \bar{u}_{h}\left(x_{h}^{\prime}\right) \geq u_{h}^{\prime}, \forall h \in \mathcal{H}\right\}$. By Point 2 of Assumption 1, the set $U_{r}$ is non-empty. Fix $\left(u_{h}^{\prime}\right)_{h \in \mathcal{H}} \in U(r)$ and consider the maximization problem

$$
\begin{array}{ll}
\max _{(x, y) \in \mathbb{R}_{++}^{C+} \times \mathbb{R}^{C J}} & \bar{u}_{1}\left(x_{1}\right) \\
\text { subject to } & \left\{\begin{array}{l}
\bar{t}_{j}\left(y_{j}\right) \leq 0, \forall j \in \mathcal{J} \\
\bar{u}_{h}\left(x_{h}\right) \geq u_{h}^{\prime}, \forall h \in \mathcal{H} \\
\sum_{h \in \mathcal{H}} x_{h}-\sum_{j \in \mathcal{J}} y_{j} \leq r
\end{array}\right.
\end{array}
$$

Step 1. Problem (12) has at least a solution. Let $K$ be the set determined by the constraints of problem (12). $K$ is non-empty since $\left(u_{h}^{\prime}\right)_{h \in \mathcal{H}} \in U_{r}$. We claim that $K$ is compact. Define the set $N:=\left\{(x, y) \in \mathbb{R}_{++}^{C H} \times \mathbb{R}^{C J}: \bar{u}_{h}\left(x_{h}\right) \geq\right.$ $\left.u_{h}^{\prime}, \forall h \in \mathcal{H}\right\}$ and notice that $K=N \cap A(\bar{x}, \bar{y} ; r)$. Then, $K$ is bounded by Lemma 4. Furthermore, $K$ is a closed set included in $\mathbb{R}_{++}^{C H} \times \mathbb{R}^{C J}$ by Point 4 of Assumption 5 and the continuity of the functions $\bar{u}_{h}$ and $\bar{t}_{j}$. The theorem of Weierstrass implies that problem (12) has at least a solution.

Step 2. Let $(\widetilde{x}, \widetilde{y})$ be a solution to problem (12). Then, $(\widetilde{x}, \widetilde{y})$ solves the fol- 
lowing problem and it is a Pareto optimal allocation of the economy $\bar{E}$.

$$
\begin{array}{ll}
\max _{(x, y) \in \mathbb{R}_{++}^{C H} \times \mathbb{R}^{C J}} & \bar{u}_{1}\left(x_{1}\right) \\
\text { subject to } & \left\{\begin{array}{l}
-\bar{t}_{j}\left(y_{j}\right) \geq 0, \forall j \in \mathcal{J} \\
\bar{u}_{h}\left(x_{h}\right)-\bar{u}_{h}\left(\widetilde{x}_{h}\right) \geq 0, \forall h \neq 1 \\
r-\sum_{h \in \mathcal{H}} x_{h}+\sum_{j \in \mathcal{J}} y_{j} \geq 0
\end{array}\right.
\end{array}
$$

Let $\widetilde{K}$ be the set determined by the constraints of problem $(13),(\widetilde{x}, \widetilde{y})$ obviously belongs to $\widetilde{K}$. Consider now $(x, y) \in \widetilde{K}$ and remind that $K$ is the set determined by the constraints of problem (12). If $\bar{u}_{1}\left(x_{1}\right) \geq u_{1}^{\prime}$, then $(x, y) \in K$ and so $\bar{u}_{1}\left(\widetilde{x}_{1}\right) \geq \bar{u}_{1}\left(x_{1}\right)$. If $\bar{u}_{1}\left(x_{1}\right)<u_{1}^{\prime}$, then $\bar{u}_{1}\left(\widetilde{x}_{1}\right)>\bar{u}_{1}\left(x_{1}\right)$ since $\bar{u}_{1}\left(\widetilde{x}_{1}\right) \geq u_{1}^{\prime}$. Thus, $(\widetilde{x}, \widetilde{y})$ solves problem (13). Now, suppose by contradiction that $(\widetilde{x}, \widetilde{y})$ is not a Pareto optimal allocation of $\bar{E}$. Then, there is another allocation $(\widehat{x}, \widehat{y}) \in \mathbb{R}_{++}^{C H} \times \mathbb{R}^{C J}$ such that $\bar{t}_{j}\left(\widehat{y}_{j}\right) \leq 0$ for all $j, \sum_{h \in \mathcal{H}} \widehat{x}_{h} \leq r+\sum_{j \in \mathcal{J}} \widehat{y}_{j}$, $\bar{u}_{h}\left(\widehat{x}_{h}\right) \geq \bar{u}_{h}\left(\widetilde{x}_{h}\right)$ for all $h$, and $\bar{u}_{k}\left(\widehat{x}_{k}\right)>\bar{u}_{k}\left(\widetilde{x}_{k}\right)$ for some $k \in \mathcal{H}$. If $k=1$, then we get a contradiction since $(\widetilde{x}, \widetilde{y})$ solves problem (13). If $k \neq 1$, using the continuity of $\bar{u}_{k}$, there exists $\varepsilon>0$ such that $\bar{u}_{k}\left(\widehat{x}_{k}-\varepsilon \mathbf{1}^{c}\right)>\bar{u}_{k}\left(\widetilde{x}_{k}\right)$, where the vector $\mathbf{1}^{c} \in \mathbb{R}_{+}^{C}$ has all the components equal to 0 except the component $c$ which is equal to 1 . Consider the allocation $(x, y)$ defined by $x_{1}:=\widehat{x}_{1}+\varepsilon \mathbf{1}^{c}$, $x_{k}:=\widehat{x}_{k}-\varepsilon \mathbf{1}^{c}, x_{h}:=\widehat{x}_{h}$ for all $h \neq 1, h \neq k$, and $y_{j}:=\widehat{y}_{j}$ for all $j$. We have that $(x, y) \in \widetilde{K}$ and $\bar{u}_{1}\left(x_{1}\right)>\bar{u}_{1}\left(\widetilde{x}_{1}\right)$ since $\bar{u}_{1}$ is strictly increasing. Then, once again we get a contradiction since $(\widetilde{x}, \widetilde{y})$ solves problem $(13)$.

Step 3. There exists $(\widetilde{\beta}, \widetilde{\theta}, \widetilde{\gamma}):=\left(\left(\widetilde{\beta}_{j}\right)_{j \in \mathcal{J}},\left(\widetilde{\theta}_{h}\right)_{h \neq 1}, \widetilde{\gamma}\right) \in \mathbb{R}_{++}^{J} \times \mathbb{R}_{++}^{H-1} \times \mathbb{R}_{++}^{C}$ such that $(\widetilde{x}, \widetilde{y}, \widetilde{\beta}, \widetilde{\theta}, \widetilde{\gamma})$ is the unique solution to system $(6)$.

We first prove the existence of $(\widetilde{\beta}, \widetilde{\theta}, \widetilde{\gamma})$, afterwards we show the uniqueness of $(\widetilde{x}, \widetilde{y}, \widetilde{\beta}, \widetilde{\theta}, \widetilde{\gamma})$.

Existence of $(\widetilde{\beta}, \widetilde{\theta}, \widetilde{\gamma}) \gg 0$. By Step 2, $(\widetilde{x}, \widetilde{y})$ solves problem (13). The KKT conditions associated with problem (13) are given by

$$
\left\{\begin{array}{l}
D_{x_{1}} \bar{u}_{1}\left(x_{1}\right)=\gamma, \forall h \neq 1: \theta_{h} D_{x_{h}} \bar{u}_{h}\left(x_{h}\right)=\gamma \text { and } \theta_{h}\left(\bar{u}_{h}\left(x_{h}\right)-\bar{u}_{h}\left(\widetilde{x}_{h}\right)\right)=0, \\
\forall j \in \mathcal{J}: \gamma=\beta_{j} D_{y_{j}} \bar{t}_{j}\left(y_{j}\right) \text { and } \beta_{j}\left(-\bar{t}_{j}\left(y_{j}\right)\right)=0, \forall c \in \mathcal{C}: \gamma^{c}\left(r^{c}-\sum_{h \in \mathcal{H}} x_{h}^{c}+\sum_{j \in \mathcal{J}} y_{j}^{c}\right)=0
\end{array}\right.
$$

where $(\beta, \theta, \gamma):=\left(\left(\beta_{j}\right)_{j \in \mathcal{J}},\left(\theta_{h}\right)_{h \neq 1},\left(\gamma^{c}\right)_{c \in \mathcal{C}}\right) \in \mathbb{R}_{+}^{J} \times \mathbb{R}_{+}^{H-1} \times \mathbb{R}_{+}^{C}$ are the Lagrange multipliers associated with the constraint functions of problem (13). We first claim that KKT conditions are necessary conditions to solve problem (13). It is enough to verify that the Jacobian matrix associated with the constraint functions of problem (13) has full row rank equal to $N:=J+(H-1)+C$. 
For every firm $j$, fix a commodity $c(j) \in \mathcal{C}$. The matrix given below is the $N \times N$ square sub-matrix which is obtained by considering the partial derivatives of the constraint functions with respect to $\left(\left(y_{j}^{c(j)}\right)_{j \in \mathcal{J}},\left(x_{h}^{1}\right)_{h \neq 1}, x_{1}\right)$. Point 4 of Assumption 1 and Point 2 of Assumption 5 imply that the determinant of this square sub-matrix is different from zero, which complete the proof of the claim. ${ }^{20}$

$$
\left[\begin{array}{ccccccc}
-D_{y_{1}^{c(1)}} \bar{t}_{1}\left(y_{1}\right) & \ldots & 0 & 0 & \cdots & 0 & 0 \\
\vdots & \ddots & \vdots & \vdots & \ddots & \vdots & \vdots \\
0 & \ldots & -D_{y_{J}^{c(J)}} \overline{\bar{t}}_{J}\left(y_{J}\right) & 0 & \cdots & 0 & 0 \\
0 & \cdots & 0 & D_{x_{2}^{1}} \bar{u}_{2}\left(x_{2}\right) & \ldots & 0 & 0 \\
\vdots & \ddots & \vdots & \vdots & \ddots & \vdots & \vdots \\
0 & \cdots & 0 & 0 & \ldots & D_{x_{H}^{1}} \bar{u}_{H}\left(x_{H}\right) & 0 \\
{\left[1^{c(1)}\right]^{T}} & \cdots & {\left[\mathbf{1}^{c(J)}\right]^{T}} & -\left[\mathbf{1}^{1}\right]^{T} & \ldots & -\left[\mathbf{1}^{1}\right]^{T} & -I_{C}
\end{array}\right]
$$

Therefore, there exists $(\widetilde{\beta}, \widetilde{\theta}, \widetilde{\gamma}) \geq 0$ such that $(\widetilde{x}, \widetilde{y}, \widetilde{\beta}, \widetilde{\theta}, \widetilde{\gamma})$ solves system (14). Furthermore, Point 4 of Assumption 1 and Point 2 of Assumption 5 imply that all the Lagrange multipliers $(\widetilde{\beta}, \widetilde{\theta}, \widetilde{\gamma})$ must be strictly positive. Consequently, all the constraints of problem (13) are binding, and then $(\widetilde{x}, \widetilde{y}, \widetilde{\beta}, \widetilde{\theta}, \widetilde{\gamma})$ is a solution to system (6).

Uniqueness of $(\widetilde{x}, \widetilde{y}, \widetilde{\beta}, \widetilde{\theta}, \widetilde{\gamma})$. Define $\widetilde{\theta}_{1}:=1$, by equations $(1)$ and (2) of system (6), for all $h$ one gets $D_{x_{h}} \bar{u}_{h}\left(\widetilde{x}_{h}\right)=\frac{\widetilde{\gamma}}{\widetilde{\theta}_{h}}$. Then, for every $h, \widetilde{x}_{h}$ solves the maximization problem: $\max _{x_{h} \in \mathbb{R}_{++}^{C}} \bar{u}_{h}\left(x_{h}\right)$ subject to $\frac{\widetilde{\gamma}}{\widetilde{\theta}_{h}} \cdot x_{h} \leq \frac{\widetilde{\gamma}}{\widetilde{\theta}_{h}} \cdot \widetilde{x}_{h}$ because KKT are sufficient conditions to solve this problem. Thus, the uniqueness of $\widetilde{x}_{h}$ follows from the strict quasi-concavity of $\bar{u}_{h}$. Analogously, by equations (4) and (5) of system (6), $\widetilde{y}_{j}$ solves the maximization problem: $\max _{y_{j} \in \mathbb{R}^{C}} \frac{\widetilde{\gamma}}{\widetilde{\beta}_{j}} \cdot y_{j}$ subject to $\bar{t}_{j}\left(y_{j}\right) \leq 0$ for every $j$. Thus, the uniqueness of $\widetilde{y}_{j}$ follows from the continuity and the strict quasi-convexity of $\bar{t}_{j}$. Therefore, $(\widetilde{x}, \widetilde{y})$ is unique and consequently, the uniqueness of $(\widetilde{\beta}, \widetilde{\theta}, \widetilde{\gamma})$ follows from equations (1), (2) and (4) of system (6).

Proof of Proposition 12. We use the functions $\bar{u}_{h}$ and $\bar{t}_{j}$ defined in Subsection 4.1. We have already pointed out that $G(\widetilde{\xi})=0$. Let $\xi^{\prime}=\left(x^{\prime}, \lambda^{\prime}, y^{\prime}, \alpha^{\prime}, p^{\prime}\right) \in$ $\Xi$ be such that $G\left(\xi^{\prime}\right)=0$, we show that $\widetilde{\xi}=\xi^{\prime}$.

First, notice that

$$
\sum_{h \in \mathcal{H}} x_{h}^{\prime}-\sum_{j \in \mathcal{J}} y_{j}^{\prime}=\sum_{h \in \mathcal{H}} e_{h}
$$

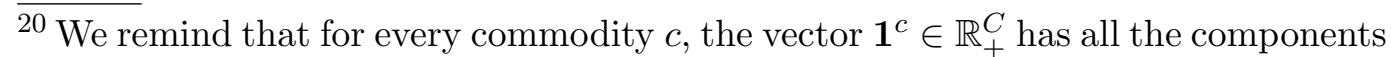
equal to 0 except the component $c$ which is equal to 1 . $I_{C}$ denotes the $C \times C$ identity matrix. 
Indeed, summing $G^{h .2}\left(\xi^{\prime}\right)=0$ over $h$, one gets $\sum_{h \in \mathcal{H}} x_{h}^{\prime}-\sum_{j \in \mathcal{J}} y_{j}^{\prime}=\sum_{h \in \mathcal{H}} \widetilde{e}_{h}$ by $G^{M}\left(\xi^{\prime}\right)=0$. Using the definition of $\widetilde{e}_{h}$ given in (7) and Proposition 11, one deduces (15).

Second, we show that

$$
\bar{u}_{h}\left(x_{h}^{\prime}\right)=\bar{u}_{h}\left(\widetilde{x}_{h}\right), \forall h \in \mathcal{H}
$$

Using the definition of $\widetilde{e}_{h}$ given in $(7)$ and $G^{h .1}\left(\xi^{\prime}\right)=G^{h .2}\left(\xi^{\prime}\right)=0, x_{h}^{\prime}$ solves the following maximization problem

$$
\begin{aligned}
& \max _{x_{h} \in \mathbb{R}_{++}^{C}} \bar{u}_{h}\left(x_{h}\right) \\
& \text { subject to } p^{\prime} \cdot x_{h} \leq p^{\prime} \cdot \widetilde{x}_{h}+\sum_{j \in \mathcal{J}} s_{j h} p^{\prime} \cdot\left(y_{j}^{\prime}-\widetilde{y}_{j}\right)
\end{aligned}
$$

because KKT are sufficient conditions to solve this problem. Analogously, from $G^{j .1}\left(\xi^{\prime}\right)=G^{j .2}\left(\xi^{\prime}\right)=0, y_{j}^{\prime}$ solves the maximization problem: $\max _{y_{j} \in \mathbb{R}^{C}} p^{\prime} \cdot y_{j}$ subject to $\bar{t}_{j}\left(y_{j}\right) \leq 0$. Notice that $\widetilde{y}_{j}$ satisfies the constraint of this problem because $G^{j .2}(\widetilde{\xi})=0$. Thus, $p^{\prime} \cdot\left(y_{j}^{\prime}-\widetilde{y}_{j}\right) \geq 0$ for all $j$, and consequently $\widetilde{x}_{h}$ belongs to the budget constraint of problem (17). Then, $\bar{u}_{h}\left(x_{h}^{\prime}\right) \geq \bar{u}_{h}\left(\widetilde{x}_{h}\right)$ for all $h$. Now, suppose that $\bar{u}_{k}\left(x_{k}^{\prime}\right)>\bar{u}_{k}\left(\widetilde{x}_{k}\right)$ for some $k \in \mathcal{H}$. From $(15)$ and $G^{j .2}\left(\xi^{\prime}\right)=0$ for all $j$, one deduces that $\left(x^{\prime}, y^{\prime}\right)$ is a feasible allocation of the production economy $\bar{E}$, and then one gets a contradiction since $(\widetilde{x}, \widetilde{y})$ is a Pareto optimal allocation of $\bar{E}$ by Proposition 11. Thus, (16) is completely proved.

Now, define $\beta^{\prime}:=\left(\beta_{j}^{\prime}\right)_{j \in \mathcal{J}}$ where $\beta_{j}^{\prime}:=\lambda_{1}^{\prime} \alpha_{j}^{\prime}$ for all $j, \theta^{\prime}:=\left(\theta_{h}^{\prime}\right)_{h \neq 1}$ where $\theta_{h}^{\prime}:=$ $\frac{\lambda_{1}^{\prime}}{\lambda_{h}^{\prime}}$ for all $h \neq 1$ and $\gamma^{\prime}:=\lambda_{1}^{\prime} p^{\prime}$. From $G^{h .1}\left(\xi^{\prime}\right)=0$ for all $h, G^{j .1}\left(\xi^{\prime}\right)=G^{j .2}\left(\xi^{\prime}\right)=$ 0 for all $j,(15)$ and (16), it is an easy matter to check that $\left(x^{\prime}, y^{\prime}, \beta^{\prime}, \theta^{\prime}, \gamma^{\prime}\right)$ solves system (6). Then, Proposition 11 implies that $(\widetilde{x}, \widetilde{y}, \widetilde{\beta}, \widetilde{\theta}, \widetilde{\gamma})=\left(x^{\prime}, y^{\prime}, \beta^{\prime}, \theta^{\prime}, \gamma^{\prime}\right)$, and consequently, one deduces that $\widetilde{\xi}=\xi^{\prime}$.

We remark that $G$ is $C^{1}$ by Point 1 of Assumptions 1 and 5. Finally, in order to show that 0 is a regular value for $G$, one proves that $D_{\xi} G(\widetilde{\xi})$ has full row rank. In this regard, we show that if $\Delta D_{\xi} G(\widetilde{\xi})=0$, then $\Delta=0$ where $\Delta:=$

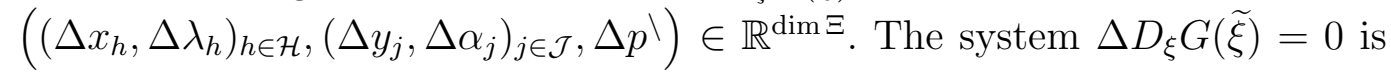


given below.

$$
\left\{\begin{array}{l}
(h .1) \quad \Delta x_{h} D_{x_{h}}^{2} \bar{u}_{h}\left(\widetilde{x}_{h}\right)-\Delta \lambda_{h} \widetilde{p}+\Delta p \backslash\left[I_{C-1} \mid 0\right]=0, \forall h \in \mathcal{H} \\
(h .2) \quad-\Delta x_{h} \cdot \widetilde{p}=0, \forall h \in \mathcal{H} \\
(j .1) \quad \sum_{h \in \mathcal{H}} \Delta \lambda_{h} s_{j h} \widetilde{p}-\widetilde{\alpha}_{j} \Delta y_{j} D_{y_{j}}^{2} \bar{t}_{j}\left(\widetilde{y}_{j}\right)-\Delta \alpha_{j} D_{y_{j}} \bar{t}_{j}\left(\widetilde{y}_{j}\right)-\Delta p \backslash\left[I_{C-1} \mid 0\right]=0, \forall j \in \mathcal{J} \\
(j .2) \quad-\Delta y_{j} \cdot D_{y_{j}} \bar{t}_{j}\left(\widetilde{y}_{j}\right)=0, \forall j \in \mathcal{J} \\
(M) \quad-\sum_{h \in \mathcal{H}} \widetilde{\lambda}_{h} \Delta x_{h}+\sum_{j \in \mathcal{J}} \Delta y_{j}^{\backslash}=0
\end{array}\right.
$$

We first prove that $\Delta x_{h}=0$ for all $h \in \mathcal{H}$. Otherwise, suppose that there is $\bar{h} \in \mathcal{H}$ such that $\Delta x_{\bar{h}} \neq 0$. The proof goes through the two following claims that contradict each others.

We first claim that $\Delta p \backslash \cdot\left(\sum_{h \in \mathcal{H}} \tilde{\lambda}_{h} \Delta x_{h}^{\backslash}\right)>0$. Multiplying $(h .1)$ by $\tilde{\lambda}_{h} \Delta x_{h}$ and summing over $h$, from $(h .2)$ we get $\sum_{h \in \mathcal{H}} \widetilde{\lambda}_{h} \Delta x_{h} D_{x_{h}}^{2} \bar{u}_{h}\left(\widetilde{x}_{h}\right)\left(\Delta x_{h}\right)=-\Delta p \backslash$. $\left(\sum_{h \in \mathcal{H}} \widetilde{\lambda}_{h} \Delta x_{h}^{\backslash}\right)$. Multiplying $G^{h .1}(\widetilde{\xi})=0$ by $\Delta x_{h}$ and using $(h .2)$, we get $\Delta x_{h}$. $D_{x_{h}} \bar{u}_{h}\left(\widetilde{x}_{h}\right)=0$ for all $h$. Therefore, Point 3 of Assumption 5 completes the proof of the claim since $\tilde{\lambda}_{h}>0$ for all $h$ and $\Delta x_{\bar{h}} \neq 0$.

Second, we claim that $\Delta p \backslash \cdot\left(\sum_{h \in \mathcal{H}} \widetilde{\lambda}_{h} \Delta x_{h}^{\backslash}\right) \leq 0$. Multiplying both sides of $G^{j .1}(\widetilde{\xi})=0$ by $\Delta y_{j}$ and using $(j .2)$, we get $\Delta y_{j} \cdot \widetilde{p}=0$. Then, multiplying $(j .1)$ by $\Delta y_{j}$ and summing over $j$, from $(j .2)$ we get $-\sum_{j \in \mathcal{J}} \widetilde{\alpha}_{j} \Delta y_{j} D_{y_{j}}^{2} \bar{t}_{j}\left(\widetilde{y}_{j}\right)\left(\Delta y_{j}\right)=$ $\Delta p \backslash \cdot \sum_{j \in \mathcal{J}} \Delta y_{j}$. Since, $\widetilde{\alpha}_{j}>0$ for all $j$, Point 3 of Assumption 1 and (j.2) imply that $\Delta p \backslash \cdot \sum_{j \in \mathcal{J}} \Delta y_{j} \leq 0$. Using $(M)$, the claim is completely proved.

Since $\widetilde{p}^{C}=1$ and $\Delta x_{h}=0$ for all $h \in \mathcal{H}$, from (h.1) we get $\Delta \lambda_{h}=0$ for all $h$, and then $\Delta p \backslash=0$. Thus, multiplying $(j .1)$ by $\Delta y_{j}$, Point 3 of Assumption 1 and $(j .2)$ imply that $\Delta y_{j}=0$. Therefore, using once again $(j .1)$, we get $\Delta \alpha_{j}=0$ by Point 4 of Assumption 1. Thus, we get $\Delta=0$.

Proof of Proposition 15. Observe that $H^{-1}(0)=\Phi^{-1}(0) \cup \Gamma^{-1}(0)$. Since the union of a finite number of compact sets is compact, it is enough to show that $\Phi^{-1}(0)$ and $\Gamma^{-1}(0)$ are compact.

Claim 1. $\Phi^{-1}(0)$ is compact.

We prove that, up to a subsequence, every sequence $\left(\xi^{\nu}, \tau^{\nu}\right)_{\nu \in \mathbb{N}} \subseteq \Phi^{-1}(0)$ 
converges to an element of $\Phi^{-1}(0)$, where $\xi^{\nu}:=\left(x^{\nu}, \lambda^{\nu}, y^{\nu}, \alpha^{\nu}, p^{\nu}\right)_{\nu \in \mathbb{N}}$. Since $\left\{\tau^{\nu}: \nu \in \mathbb{N}\right\} \subseteq[0,1]$, up to a subsequence, $\left(\tau^{\nu}\right)_{\nu \in \mathbb{N}}$ converges to some $\tau^{*} \in$ $[0,1]$. From Steps 1.1, 1.2, 1.3 and 1.4 below, up to a subsequence, $\left(\xi^{\nu}\right)_{\nu \in \mathbb{N}}$ converges to some $\xi^{*}:=\left(x^{*}, \lambda^{*}, y^{*}, \alpha^{*}, p^{*} \backslash\right) \in \Xi$. Since $\Phi$ is continuous, taking the limit, one gets $\left(\xi^{*}, \tau^{*}\right) \in \Phi^{-1}(0)$.

We remind that for every $\tau \in[0,1], e_{h}(\tau)$ is given by $(9)$.

Step 1.1. Up to a subsequence, $\left(x^{\nu}, y^{\nu}\right)_{\nu \in \mathbb{N}}$ converges to some $\left(x^{*}, y^{*}\right) \in \mathbb{R}_{+}^{C H} \times$ $\mathbb{R}^{C J}$. We show that for $r=\sum_{h \in \mathcal{H}} e_{h}$, the sequence $\left(x^{\nu}, y^{\nu}\right)_{\nu \in \mathbb{N}}$ is included in the bounded set $K(r)$ given by Lemma 4 . By $\Phi^{j .2}\left(\xi^{\nu}, \tau^{\nu}\right)=0$, for every $j$ we get

$$
t_{j}\left(y_{j}^{\nu}, \bar{y}_{-j}, \bar{x}\right)=0, \forall \nu \in \mathbb{N}
$$

Thus, the sequence $\left(y^{\nu}\right)_{\nu \in \mathbb{N}}$ is included in the set $Y(\bar{x}, \bar{y})$ given by (1). Summing $\Phi^{h .2}\left(\xi^{\nu}, \tau^{\nu}\right)=0$ over $h$, by $\Phi^{M}\left(\xi^{\nu}, \tau^{\nu}\right)=0$ we have $\sum_{h \in \mathcal{H}} x_{h}^{\nu}-\sum_{j \in \mathcal{J}} y_{j}^{\nu}=\sum_{h \in \mathcal{H}} e_{h}\left(\tau^{\nu}\right)$ for every $\nu \in \mathbb{N}$. Using the definition of $\widetilde{e}_{h}$ given by (7) and Proposition 11, one gets $\sum_{h \in \mathcal{H}} e_{h}(\tau)=r$ for every $\tau \in[0,1]$, and then $\sum_{h \in \mathcal{H}} x_{h}^{\nu}-\sum_{j \in \mathcal{J}} y_{j}^{\nu}=r$ for every $\nu \in \mathbb{N}$. Thus, $\left(x^{\nu}, y^{\nu}\right)_{\nu \in \mathbb{N}} \subseteq A(\bar{x}, \bar{y} ; r) \subseteq K(r)$. Consequently, $\left(x^{\nu}, y^{\nu}\right)_{\nu \in \mathbb{N}}$ is included in $\mathrm{cl} K(r)$ which is a compact set. Therefore, up to a subsequence, $\left(x^{\nu}, y^{\nu}\right)_{\nu \in \mathbb{N}}$ converges to some $\left(x^{*}, y^{*}\right) \in \operatorname{cl} K(r) \subseteq \mathbb{R}_{+}^{C H} \times \mathbb{R}^{C J}$, and then $\left(x^{*}, y^{*}\right) \in \mathbb{R}_{+}^{C H} \times \mathbb{R}^{C J}$.

Step 1.2. The consumption allocation $x^{*}$ is strictly positive, i.e. $x_{h}^{*} \gg 0$ for every $h \in \mathcal{H}$. By $\Phi^{h .1}\left(\xi^{\nu}, \tau^{\nu}\right)=\Phi^{h .2}\left(\xi^{\nu}, \tau^{\nu}\right)=0$ and KKT sufficient conditions, $x_{h}^{\nu}$ solves the following problem for every $\nu \in \mathbb{N}$.

$$
\begin{aligned}
& \max _{x_{h} \in \mathbb{R}_{++}^{C}} u_{h}\left(x_{h}, \bar{x}_{-h}, \bar{y}\right) \\
& \text { subject to } p^{\nu} \cdot x_{h} \leq p^{\nu} \cdot\left[\tau^{\nu} e_{h}+\left(1-\tau^{\nu}\right) \widetilde{x}_{h}\right]+p^{\nu} \cdot \sum_{j \in \mathcal{J}} s_{j h}\left(y_{j}^{\nu}-\left(1-\tau^{\nu}\right) \widetilde{y}_{j}\right)
\end{aligned}
$$

We first claim that for every $\nu \in \mathbb{N}$, the following vector

$$
\widehat{e}_{h}\left(\tau^{\nu}\right):=\tau^{\nu} e_{h}+\left(1-\tau^{\nu}\right) \widetilde{x}_{h}
$$

belongs to the budget constraint of the problem above. By $\Phi^{j .1}\left(\xi^{\nu}, \tau^{\nu}\right)=$ $\Phi^{j .2}\left(\xi^{\nu}, \tau^{\nu}\right)=0$ and KKT sufficient conditions, $y_{j}^{\nu}$ solves the following problem for every $\nu \in \mathbb{N}$.

$$
\begin{aligned}
& \max _{y_{j} \in \mathbb{R}^{C}} p^{\nu} \cdot y_{j} \\
& \text { subject to } t_{j}\left(y_{j}, \bar{y}_{-j}, \bar{x}\right) \leq 0
\end{aligned}
$$

$t_{j}\left(\widetilde{y}_{j}, \bar{y}_{-j}, \bar{x}\right)=0$ since $G^{j .2}(\widetilde{\xi})=0$, see (8). By Point 2 of Assumption 1, $t_{j}\left(0, \bar{y}_{-j}, \bar{x}\right)=0$. Then, we get $t_{j}\left(\left(1-\tau^{\nu}\right) \widetilde{y}_{j}, \bar{y}_{-j}, \bar{x}\right)<0$ since $t_{j}\left(\cdot, \bar{y}_{-j}, \bar{x}\right)$ is 
strictly quasi-convex, that is, the production plan $\left(1-\tau^{\nu}\right) \widetilde{y}_{j}$ belongs to the constraint set of problem (20). Thus, $p^{\nu} \cdot\left(y_{j}^{\nu}-\left(1-\tau^{\nu}\right) \widetilde{y}_{j}\right) \geq 0$ for every $j$, and then $p^{\nu} \cdot \sum_{j \in \mathcal{J}} s_{j h}\left(y_{j}^{\nu}-\left(1-\tau^{\nu}\right) \widetilde{y}_{j}\right) \geq 0$ which completes the proof of the claim.

Therefore, for every $\nu \in \mathbb{N}, u_{h}\left(x_{h}^{\nu}, \bar{x}_{-h}, \bar{y}\right) \geq u_{h}\left(\widehat{e}_{h}\left(\tau^{\nu}\right), \bar{x}_{-h}, \bar{y}\right)$. By Point 2 of Assumption 5, for every $\varepsilon>0$ we get $u_{h}\left(x_{h}^{\nu}+\varepsilon \mathbf{1}, \bar{x}_{-h}, \bar{y}\right)>u_{h}\left(\widehat{e}_{h}\left(\tau^{\nu}\right), \bar{x}_{-h}, \bar{y}\right)$ where $1:=(1, \ldots, 1) \in \mathbb{R}_{++}^{C}$. Taking the limit over $\nu$ and using Point 1 of Assumption 5, we get $u_{h}\left(x_{h}^{*}+\varepsilon \mathbf{1}, \bar{x}_{-h}, \bar{y}\right) \geq u_{h}\left(\widehat{e}_{h}\left(\tau^{*}\right), \bar{x}_{-h}, \bar{y}\right)$ for every $\varepsilon>0$. Then, $x_{h}^{*}$ belongs to the closure of the upper counter set of $\left(\widehat{e}_{h}\left(\tau^{*}\right), \bar{x}_{-h}, \bar{y}\right)$, which is included in $\mathbb{R}_{++}^{C}$ by Point 4 of Assumption 5. Thus, $x_{h}^{*} \gg 0$.

Step 1.3. Up to a subsequence, $\left(\lambda^{\nu}, p^{\nu}\right)_{\nu \in \mathbb{N}}$ converges to some $\lambda^{*} \in \mathbb{R}_{++}^{H} \times$ $\mathbb{R}_{++}^{C-1}$. The proof is similar to the proof of Step 2.3.

Step 1.4. Up to a subsequence, $\left(\alpha^{\nu}\right)_{\nu \in \mathbb{N}}$ converges to some $\alpha^{*} \in \mathbb{R}_{++}^{J}$. The proof is similar to the proof of Step 2.4 .

Claim 2. $\Gamma^{-1}(0)$ is compact.

Let $\left(\xi^{\nu}, \tau^{\nu}\right)_{\nu \in \mathbb{N}}$ be a sequences in $\Gamma^{-1}(0)$. As in Claim $1,\left(\tau^{\nu}\right)_{\nu \in \mathbb{N}}$ converges to $\tau^{*} \in[0,1]$. From Steps 2.1, 2.2, 2.3 and 2.4 below, up to a subsequence, $\left(\xi^{\nu}\right)_{\nu \in \mathbb{N}}$ converges to an element $\xi^{*}:=\left(x^{*}, \lambda^{*}, y^{*}, \alpha^{*}, p^{*}\right) \in \Xi$. Since $\Gamma$ is a continuous function, taking limit, we get $\left(\xi^{*}, \tau^{*}\right) \in \Gamma^{-1}(0)$.

We remind that for every $\tau \in[0,1], x(\tau)$ and $y(\tau)$ are given by $(9)$.

Step 2.1. Up to a subsequence, $\left(x^{\nu}, y^{\nu}\right)_{\nu \in \mathbb{N}}$ converges to some $\left(x^{*}, y^{*}\right) \in \mathbb{R}_{+}^{C H} \times$ $\mathbb{R}^{C J}$. We show that for $r=\sum_{h \in \mathcal{H}} e_{h}$, the sequence $\left(x^{\nu}, y^{\nu}\right)_{\nu \in \mathbb{N}}$ is included in the bounded set $K(r)$ given by Lemma 4 . Then, one completes the proof as in Step 1.1. By $\Gamma^{j .2}\left(\xi^{\nu}, \tau^{\nu}\right)=0$, for every $j$ we have that

$$
t_{j}\left(y_{j}^{\nu}, y_{-j}^{\nu}\left(\tau^{\nu}\right), x^{\nu}\left(\tau^{\nu}\right)\right)=0, \forall \nu \in \mathbb{N}
$$

Thus, for every $\nu \in \mathbb{N}$, the production allocation $y^{\nu}$ belongs to the set $Y\left(x^{\nu}\left(\tau^{\nu}\right), y^{\nu}\left(\tau^{\nu}\right)\right)$ given by (1). Now, summing $\Gamma^{h .2}\left(\xi^{\nu}, \tau^{\nu}\right)=0$ over $h$, by $\Gamma^{M}\left(\xi^{\nu}, \tau^{\nu}\right)=0$, we get $\sum_{h \in \mathcal{H}} x_{h}^{\nu}-\sum_{j \in \mathcal{J}} y_{j}^{\nu}=r$. Then, for every $\nu \in \mathbb{N}$, the allocation $\left(x^{\nu}, y^{\nu}\right)$ belongs to the set $A\left(x^{\nu}\left(\tau^{\nu}\right), y^{\nu}\left(\tau^{\nu}\right) ; r\right) \subseteq K(r)$, and consequently, $\left(x^{\nu}, y^{\nu}\right)_{\nu \in \mathbb{N}} \subseteq K(r)$.

Step 2.2. The consumption allocation $x^{*}$ is strictly positive, i.e. $x_{h}^{*} \gg 0$ for every $h \in \mathcal{H}$. The argument is similar to the one used in Step 1.2 except for the last part which is quite different due to the presence of consumption externalities in the utility functions. For this reason, at the end of this step, we need both the continuity of $u_{h}$ on $\mathbb{R}_{++}^{C} \times \mathbb{R}_{+}^{C(H-1)} \times \mathbb{R}^{C J}$ and Point 4 of Assumption 5 for all consumption externalities in $\mathbb{R}_{+}^{C(H-1)}$ or, alternatively, 
the continuity of $u_{h}$ on $\mathbb{R}_{++}^{C} \times \mathbb{R}_{++}^{C(H-1)} \times \mathbb{R}^{C J}$, Point 4 of Assumption 5 only for consumption externalities in $\mathbb{R}_{++}^{C(H-1)}$, and Point 5 of Section 2.

First, according to $\Gamma^{h .1}\left(\xi^{\nu}, \tau^{\nu}\right)=\Gamma^{h .2}\left(\xi^{\nu}, \tau^{\nu}\right)=0$, replace problem (18) with the following problem

$$
\begin{aligned}
& \max _{x_{h} \in \mathbb{R}_{++}^{C}} u_{h}\left(x_{h}, x_{-h}^{\nu}\left(\tau^{\nu}\right), y^{\nu}\left(\tau^{\nu}\right)\right) \\
& \text { subject to } p^{\nu} \cdot x_{h} \leq p^{\nu} \cdot e_{h}+p^{\nu} \cdot \sum_{j \in \mathcal{J}} s_{j h} y_{j}^{\nu}
\end{aligned}
$$

and replace $\widehat{e}_{h}\left(\tau^{\nu}\right)$ given by (19) with $\widehat{e}_{h}\left(\tau^{\nu}\right):=e_{h}$. Second, according to $\Gamma^{j .1}\left(\xi^{\nu}, \tau^{\nu}\right)=\Gamma^{j .2}\left(\xi^{\nu}, \tau^{\nu}\right)=0$, replace problem (20) with the following problem

$$
\begin{aligned}
& \max _{y_{j} \in \mathbb{R}^{C}} p^{\nu} \cdot y_{j} \\
& \text { subject to } t_{j}\left(y_{j}, y_{-j}^{\nu}\left(\tau^{\nu}\right), x^{\nu}\left(\tau^{\nu}\right)\right) \leq 0
\end{aligned}
$$

Third, one follows the same strategy as for Step 1.2. For every $\nu \in \mathbb{N}$, $u_{h}\left(x_{h}^{\nu}, x_{-h}^{\nu}\left(\tau^{\nu}\right), y^{\nu}\left(\tau^{\nu}\right)\right) \geq u_{h}\left(e_{h}, x_{-h}^{\nu}\left(\tau^{\nu}\right), y^{\nu}\left(\tau^{\nu}\right)\right)$. Notice that $x_{-h}^{\nu}\left(\tau^{\nu}\right)$ belongs to $\mathbb{R}_{++}^{C(H-1)}$, because $x_{-h}^{\nu}\left(\tau^{\nu}\right)=\tau^{\nu} x_{-h}^{\nu}+\left(1-\tau^{\nu}\right) \bar{x}_{-h}$, and $x_{-h}^{\nu}$ and $\bar{x}_{-h}$ are both strictly positive. Then, by Point 2 of Assumption 5 , for every $\nu \in \mathbb{N}$ and for every $\varepsilon>0$ we get

$$
u_{h}\left(x_{h}^{\nu}+\varepsilon \mathbf{1}, x_{-h}^{\nu}\left(\tau^{\nu}\right), y^{\nu}\left(\tau^{\nu}\right)\right)>u_{h}\left(e_{h}, x_{-h}^{\nu}\left(\tau^{\nu}\right), y^{\nu}\left(\tau^{\nu}\right)\right)
$$

Now, take the limit over $\nu$. Differently from Step 1.2, we have the two following cases: $x_{-h}^{*}\left(\tau^{*}\right) \gg 0$ or $x_{-h}^{*}\left(\tau^{*}\right) \in \mathrm{Bd}\left(\mathbb{R}_{++}^{C(H-1)}\right)$. The second case may happen here, but not in Step 1.2, because now there are consumption externalities in the utility functions. Indeed, if $\left(\tau^{\nu}\right)_{\nu \in \mathbb{N}}$ converges to $\tau^{*}=1$, then $x_{-h}^{*}\left(\tau^{*}\right)=$ $x_{-h}^{*}$ which a priori is not necessarily strictly positive.

Suppose that $x_{-h}^{*}\left(\tau^{*}\right) \gg 0$. Using (22) and Point 1 of Assumption 5, we get $u_{h}\left(x_{h}^{*}+\varepsilon \mathbf{1}, x_{-h}^{*}\left(\tau^{*}\right), y^{*}\left(\tau^{*}\right)\right) \geq u_{h}\left(e_{h}, x_{-h}^{*}\left(\tau^{*}\right), y^{*}\left(\tau^{*}\right)\right)$ for every $\varepsilon>0$. Then, $x_{h}^{*}$ is strictly positive, because it belongs to the closure of the upper counter set of $\left(e_{h}, x_{-h}^{*}\left(\tau^{*}\right), y^{*}\left(\tau^{*}\right)\right)$, which is included in $\mathbb{R}_{++}^{C}$ by Point 4 of Assumption 5. Suppose now that $x_{-h}^{*}\left(\tau^{*}\right) \in \operatorname{Bd}\left(\mathbb{R}_{++}^{C(H-1)}\right)$. Under Point 1 and Point 4 of Assumption 5, the previous argument still holds true. Alternatively, if $u_{h}$ does not satisfy BC whenever consumption externalities converge to zero for some commodities, one uses Point 5 in Section 2 as follows. By Point 5 and (22), there exists $\delta>0$ such that for every $\nu \in \mathbb{N}$ and for every $\varepsilon>0$ one gets

$$
u_{h}\left(x_{h}^{\nu}+\varepsilon \mathbf{1}, x_{-h}^{\nu}\left(\tau^{\nu}\right)+\delta \mathbf{1}, y^{\nu}\left(\tau^{\nu}\right)\right) \geq u_{h}\left(e_{h}, x_{-h}^{\nu}\left(\tau^{\nu}\right)+\delta \mathbf{1}, y^{\nu}\left(\tau^{\nu}\right)\right)
$$

Take the limit over $\nu$ and remark that $x_{-h}^{*}\left(\tau^{*}\right)+\delta \mathbf{1} \gg 0$. Consequently, one gets $u_{h}\left(x_{h}^{*}+\varepsilon \mathbf{1}, x_{-h}^{*}\left(\tau^{*}\right)+\delta \mathbf{1}, y^{*}\left(\tau^{*}\right)\right) \geq u_{h}\left(e_{h}, x_{-h}^{*}\left(\tau^{*}\right)+\delta \mathbf{1}, y^{*}\left(\tau^{*}\right)\right)$ for every 
$\varepsilon>0$, because in this case $u_{h}$ is required to be continuous on $\mathbb{R}_{++}^{C} \times \mathbb{R}_{++}^{C(H-1)} \times$ $\mathbb{R}^{C J}$. Then, $x_{h}^{*}$ is strictly positive since it belongs to the closure of the upper counter set of $\left(e_{h}, x_{-h}^{*}\left(\tau^{*}\right)+\delta \mathbf{1}, y^{*}\left(\tau^{*}\right)\right)$, which is included in $\mathbb{R}_{++}^{C}$ because $x_{-h}^{*}\left(\tau^{*}\right)+\delta 1 \gg 0$ and because in this case Point 4 of Assumption 5 is required only for consumption externalities in $\mathbb{R}_{++}^{C(H-1)}$.

Step 2.3. Up to a subsequence, $\left(\lambda^{\nu}, p^{\nu} \backslash\right)_{\nu \in \mathbb{N}}$ converges to some $\left(\lambda^{*}, p^{*} \backslash\right) \in$ $\mathbb{R}_{++}^{H} \times \mathbb{R}_{++}^{C-1}$. By $\Gamma^{h .1}\left(\xi^{\nu}, \tau^{\nu}\right)=0$, fixing commodity $C$, for every $\nu \in \mathbb{N}$ we have $\lambda_{h}^{\nu}=D_{x_{h}^{C}} u_{h}\left(x_{h}^{\nu}, x_{-h}^{\nu}\left(\tau^{\nu}\right), y^{\nu}\left(\tau^{\nu}\right)\right)$. Taking the limit, by Points 1 and 2 of Assumption 5, we get $\lambda_{h}^{*}:=D_{x_{h}^{C}} u_{h}\left(x_{h}^{*}, x_{-h}^{*}\left(\tau^{*}\right), y^{*}\left(\tau^{*}\right)\right)>0$.

By $\Gamma^{h .1}\left(\xi^{\nu}, \tau^{\nu}\right)=0$, for all commodity $c \neq C$ and for all $\nu \in \mathbb{N}$ we have $p^{\nu c}=$ $\frac{D_{x_{h}^{c}} u_{h}\left(x_{h}^{\nu}, x_{-h}^{\nu}\left(\tau^{\nu}\right), y^{\nu}\left(\tau^{\nu}\right)\right)}{\lambda_{h}^{\nu}}$. Taking the limit, by Points 1 and 2 of Assumption 5 , we get $p^{* c}:=\frac{D_{x_{h}^{c}} u_{h}\left(x_{h}^{*}, x_{-h}^{*}\left(\tau^{*}\right), y^{*}\left(\tau^{*}\right)\right)}{\lambda_{h}^{*}}>0$. Therefore, $p^{* \backslash} \gg 0$.

Step 2.4. Up to a subsequence, $\left(\alpha^{\nu}\right)_{\nu \in \mathbb{N}}$ converges to some $\alpha^{*} \in \mathbb{R}_{++}^{J}$. For every firm $j$, fix a commodity $c(j) \in \mathcal{C}$. By $\Gamma^{j .1}\left(\xi^{\nu}, \tau^{\nu}\right)=0$, for every $\nu \in \mathbb{N}$ we have that

$$
\alpha_{j}^{\nu}=\frac{p^{\nu c(j)}}{D_{y_{j}^{c(j)}} t_{j}\left(y_{j}^{\nu}, y_{-j}^{\nu}\left(\tau^{\nu}\right), x^{\nu}\left(\tau^{\nu}\right)\right)}
$$

which is strictly positive by Point 4 of Assumption 1. Taking the limit, by Points 1 and 4 of Assumption 1 , we get $\alpha_{j}^{*}:=\frac{p^{* c(j)}}{D_{y_{j}^{c(j)}} t_{j}\left(y_{j}^{*}, y_{-j}^{*}\left(\tau^{*}\right), x^{*}\left(\tau^{*}\right)\right)}>0$.

\section{Appendix}

We introduce a definition of the degree modulo 2 for continuous functions, see Appendix B in Geanakoplos and Shafer (1990), and Chapter 7 in Villanacci et al. (2002).

Let $M$ and $N$ be two $C^{2}$ manifolds of the same dimension contained in euclidean spaces. Let $\mathcal{A}$ be the set of triples $(f, M, y)$ where

(1) $f: M \rightarrow N$ is a continuous function,

(2) $y \in N$ and $f^{-1}(y)$ is compact.

Theorem 16 There exists a unique function, called degree modulo 2 and denoted by $\operatorname{deg}_{2}: \mathcal{A} \rightarrow\{0,1\}$ such that 
(1) (Normalisation) $\operatorname{deg}_{2}\left(i d_{M}, M, y\right)=1$

where $y \in M$ and $i d_{M}$ denotes the identity of $M$.

(2) (Non-triviality) If $(f, M, y) \in \mathcal{A}$ and $\operatorname{deg}_{2}(f, M, y)=1$, then $f^{-1}(y) \neq \emptyset$.

(3) (Excision) If $(f, M, y) \in \mathcal{A}$ and $U$ is an open subset of $M$ such that $f^{-1}(y) \subseteq U$, then

$$
\operatorname{deg}_{2}(f, M, y)=\operatorname{deg}_{2}(f, U, y)
$$

(4) (Additivity) If $(f, M, y) \in \mathcal{A}$ and $U_{1}$ and $U_{2}$ are open and disjoint subsets of $M$ such that $f^{-1}(y) \subseteq U_{1} \cup U_{2}$, then

$$
\operatorname{deg}_{2}(f, M, y)=\operatorname{deg}_{2}\left(f, U_{1}, y\right)+\operatorname{deg}_{2}\left(f, U_{2}, y\right)
$$

(5) (Local constantness) If $(f, M, y) \in \mathcal{A}$ and $U$ is an open subset of $M$ with compact closure such that $f^{-1}(y) \subseteq U$, then there is an open neighborhood $V$ of $y$ in $N$ such that for every $y^{\prime} \in V$,

$$
\operatorname{deg}_{2}\left(f, U, y^{\prime}\right)=\operatorname{deg}_{2}(f, U, y)
$$

(6) (Homotopy invariance) Let $L:(z, \tau) \in M \times[0,1] \rightarrow L(z, \tau) \in N$ be a continuous homotopy. If $y \in N$ and $L^{-1}(y)$ is compact, then

$$
\operatorname{deg}_{2}\left(L_{0}, U, y\right)=\operatorname{deg}_{2}\left(L_{1}, U, y\right)
$$

where $L_{0}:=L(\cdot, 0): M \rightarrow N$ and $L_{1}:=L(\cdot, 1): M \rightarrow N$.

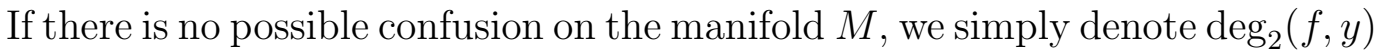
the degree modulo 2 of the triple $(f, M, y)$.

As stated in the following proposition, in the case of regular values of a $C^{1}$ function, the degree modulo 2 is computed using the residue class modulo 2.

Proposition 17 If $(g, M, y) \in \mathcal{A}, g$ is a $C^{1}$ function and $y$ is a regular value of $g$ (i.e., for all $z^{*} \in g^{-1}(y)$, the differential mapping $D g\left(z^{*}\right)$ is onto), then $g^{-1}(y)$ is finite (possibly empty) and the degree modulo 2 of $g$ is given by

$$
\operatorname{deg}_{2}(g, M, y)=\left[\# g^{-1}(y)\right]_{2}= \begin{cases}0 & \text { if } \# g^{-1}(y) \text { is even } \\ 1 & \text { if } \# g^{-1}(y) \text { is odd }\end{cases}
$$

\section{References}

Arrow, K.J., Hahn, F., 1971. General Competitive Analysis. San Francisco: Holden-Day.

Balasko, Y., 2015. Wealth concerns and equilibrium, Journal of Mathematical Economics 59, 92-101. 
Bonnisseau, J.-M., 2003. Regular economies with non-ordered preferences, Journal of Mathematical Economics 39, 153-174.

Bonnisseau, J.-M., del Mercato, E. L., 2008. General consumption constraints and regular economies, Journal of Mathematical Economics 44, 1286-1301.

Bonnisseau, J.-M., del Mercato, E. L., 2010. Externalities, consumption constraints and regular economies, Economic Theory 44, 123-147.

Bonnisseau, J.-M., Médecin, J.-P., 2001. Existence of marginal pricing equilibria in economies with externalities and non-convexities, Journal of Mathematical Economics 36, 271-294.

Debreu, G., 1959. Theory of Value. Yale University Press.

Ericson, R.E., Kung, F.-C., 2015. Fundamental non-convexities and externalities: A differentiable approach, The B.E. Journal of Theoretical Economics 15(1), 49-62.

del Mercato, E. L., 2006. Existence of competitive equilibria with externalities: A differential viewpoint, Journal of Mathematical Economics 42, 525-543.

del Mercato, E. L., Platino, V., forthcoming 2017. On the regularity of smooth production economies with externalities: competitive equilibrium à la Nash, Economic Theory.

Geanakoplos, J., Shafer, W., 1990. Solving systems of simultaneous equations in economics, Journal of Mathematical Economics 19, 69-93.

Kung, F.-C., 2008. Voluntary contributions to multiple public goods in a production economy with widespread externalities, Journal of Mathematical Economics 44, 1364-1378.

Laffont, J.-J., 1988. Fundamentals of Public Economics. The MIT Press Cambridge.

Laffont, J.-J., Laroque, G., 1972. Effets externes et théorie de l'équilibre général, Cahiers du Séminaire d'Econométrie 14, CNRS, Paris, France, 1-48.

Mandel, A., 2008. An index formula for production economies with externalities, Journal of Mathematical Economics 44, 1385-1397.

Mas-Colell, A., 1985. The Theory of General Economic Equilibrium. A Differentiable Approach. Cambridge University Press.

Mas-Colell, A., Whinston, M. D., Green, J. R., 1995. Microeconomic Theory. Oxford University Press.

Milnor, J. W., 1965. Topology from the Differentiable Viewpoint. The University Press of Virginia.

Smale, S., 1974. Global analysis and economics. II A: Extension of a theorem of Debreu, Journal of Mathematical Economics 1, 1-14.

Villanacci, A., Carosi, L., Benevieri, P., Battinelli, A., 2002. Differential Topology and General Equilibrium with Complete and Incomplete Markets. Kluwer Academic Publishers.

Villanacci, A., Zenginobuz, U., 2005. Existence and regularity of equilibria in a general equilibrium model with private provision of a public good, Journal of Mathematical Economics 41, 617-636. 